TRANSACTIONS OF THE

AMERICAN MATHEMATICAL SOCIETY

Volume 363, Number 11, November 2011, Pages 5847-5870

S 0002-9947(2011)05234-7

Article electronically published on June 2, 2011

\title{
LABELED TREES AND LOCALIZED AUTOMORPHISMS OF THE CUNTZ ALGEBRAS
}

\author{
ROBERTO CONTI AND WOJCIECH SZYMAŃSKI
}

\begin{abstract}
We initiate a detailed and systematic study of automorphisms of the Cuntz algebras $\mathcal{O}_{n}$ which preserve both the diagonal and the core UHFsubalgebra. A general criterion of invertibility of endomorphisms yielding such automorphisms is given. Combinatorial investigations of endomorphisms related to permutation matrices are presented. Key objects entering this analysis are labeled rooted trees equipped with additional data. Our analysis provides insight into the structure of $\operatorname{Aut}\left(\mathcal{O}_{n}\right)$ and leads to numerous new examples. In particular, we completely classify all such automorphisms of $\mathcal{O}_{2}$ for the permutation unitaries in $\otimes^{4} M_{2}$. We show that the subgroup of $\operatorname{Out}\left(\mathcal{O}_{2}\right)$ generated by these automorphisms contains a copy of the infinite dihedral group $\mathbb{Z} \rtimes \mathbb{Z}_{2}$.
\end{abstract}

'Nel mezzo del cammin di nostra vita

mi ritrovai per una selva oscura,

ché la diritta via era smarrita.'

Dante Alighieri, La Divina Commedia, Inferno

'Macbeth shall never vanquished be, until

great Birnam wood to high Dunsinane hill

shall come against him.'

William Shakespeare, Macbeth

\section{Contents}

1. Introduction 5848

2. Setup 5849

3. Searching for automorphisms 5

3.1. Invertibility of localized endomorphisms

3.2. Automorphisms of the diagonal 5854

4. Applications of labeled trees to the search for automorphisms

4.1. Upper left corner $[b] \quad 5855$

4.2. Effect of inner automorphisms 5858

4.3. Lower right corner $[d] \quad 5859$

4.4. A characterization of automorphisms in $\lambda\left(\mathcal{P}_{n}\right)^{-1} \quad 5860$

5. Applications of labeled trees to automorphisms of $\mathcal{O}_{2}$

Received by the editors September 8, 2008 and, in revised form, October 15, 2009.

2010 Mathematics Subject Classification. Primary 46L40, 46L05, 37B10.

Key words and phrases. Cuntz algebra, endomorphism, automorphism, Cartan subalgebra, core UHF-subalgebra, normalizer, permutation, tree. 
5.1. Case of $\mathcal{P}_{2}^{2} \quad 5860$

5.2. Case of $\mathcal{P}_{2}^{3} \quad 5861$

5.3. Case of $\mathcal{P}_{2}^{4} \quad 5861$

6. Tabulated results 5865

7. Concluding remarks 5866

8. Appendix 5867

Acknowledgements 5869

References $\quad 5869$

\section{INTRODUCTION}

In recent years endomorphisms of Cuntz algebras have received a lot of attention and have been deeply investigated from the point of view of Jones' index theory and sector theory [10, 12, 17, 4, 13, 3, 8, 14, 15. In these theories, emphasis is often placed on proper endomorphisms rather than automorphisms. However, automorphisms of Cuntz algebras have also been studied, sometimes in connection with the classification of group actions (for example, see [1, 6, 19, 16, 11, 20]). In the present paper, our main interest lies in the classification of a special class of localized automorphisms of $\mathcal{O}_{n}$.

In his beautiful paper [6], Joachim Cuntz initiated systematic investigations of the automorphism group of $\mathcal{O}_{n}$. In particular, he showed that the group of those automorphisms which preserve the diagonal subalgebra contains a maximal abelian normal subgroup whose quotient (the Weyl group) is discrete. Restricting even further to those automorphisms which preserve both the diagonal and the core UHFsubalgebra one finds even nicer structure. Thus Cuntz suggested that classification of all elements of this restricted Weyl group 'is a combinatorial problem, and should be possible'. By now thirty years have passed and this classification has not been achieved, nor is it even in sight. Presumably, this is due not to lack of interest of high power researchers in this exciting problem but rather to the great difficulties involved. It appears that there are two sources of these difficulties.

First, as demonstrated by Cuntz [6], automorphisms of $\mathcal{O}_{n}$ are best seen as a special class of endomorphisms. The latter are in a one-to-one correspondence with unitary elements of $\mathcal{O}_{n}$ via a certain natural correspondence $\mathcal{U}\left(\mathcal{O}_{n}\right) \ni u \mapsto \lambda_{u} \in$ $\operatorname{End}\left(\mathcal{O}_{n}\right)$. The problem is that in general there is no easy way of verifying which unitaries $u$ give rise to invertible endomorphisms (i.e. automorphisms) $\lambda_{u}$. In the present article we provide a remedy to this problem for a large class of endomorphisms related to unitary matrices in $M_{n^{k}}(\mathbb{C})$ contained in the UHF-subalgebra (see Theorem 3.2, below, which relies on a combination of results in [4] and 26]).

Second, again as shown by Cuntz [6], analysis of the Weyl group reduces to endomorphisms $\lambda_{u}$ corresponding to unitaries $u$ in the normalizer of the diagonal. Thanks to Power's work [21] the structure of this normalizer is well understood. In the case of a restricted Weyl group everything boils down to analysis of endomorphisms corresponding to permutation unitaries in $M_{n^{k}}(\mathbb{C})$. Thus one might hope that some straightforward combinatorial manipulations (perhaps computer aided) with permutations will bring a solution. Unfortunately, as in level $k$ there are $n^{k}$ ! such permutations, the size of the problem grows too rapidly and already for very 
small parameters exceeds the computational capacity of modern computers. For example, $4^{3} !>10^{89}$ is greater than the number of atoms in the universe.

In order to address both problems mentioned above, we develop a novel combinatorial approach to the study of permutation related endomorphisms (see Corollary 4.12, below). Its essence is the reduction of determining invertibility of $\lambda_{u}$ to a sequential process involving several steps. In this process labeled rooted trees are associated to permutations, and certain partial orders on pairs of labels are considered. These labeled trees also serve as invariants of outer automorphism classes. Our approach reduces the computational complexity so dramatically as to allow for a relatively simple solution in such cases as e.g. $n=2, k=4$. Despite $2^{4} !>2 \cdot 10^{13}$ permutations to be considered in this case, a pen and paper calculation (later verified on a computer) was possible and led to a complete classification of all permutation related automorphisms of $\mathcal{O}_{2}$ corresponding to level 4 (see Subsection 5.3. below).

In examples illustrating our theory we pay particular attention to the case of $\mathcal{O}_{2}$, since in some sense this case is the most untractable. Indeed, concrete examples of permutation related outer automorphisms of $\mathcal{O}_{n}, n \geq 3$, have already been known. It was shown recently in 26 that such automorphisms corresponding to level 2 generate in $\operatorname{Out}\left(\mathcal{O}_{n}\right)$ a group containing the free product $\mathbb{Z}_{3} * \mathbb{Z}_{2}$. On the other hand, precious little has been known until now about permutation related automorphisms of $\mathcal{O}_{2}$. To the best of our knowledge, the only known example of an outer automorphism of $\mathcal{O}_{2}$ of this type was Archbold's flip-flop [1]. Our results explain why this was so. Namely, new outer automorphisms of $\mathcal{O}_{2}$ appear only in level 4 , and to find them one has to sift through more than $2 \cdot 10^{13}$ permutations.

Our paper is organized as follows. In Section 2, we set up notation and present basic structural results about diagonal preserving automorphisms of $\mathcal{O}_{n}$ which follow more or less directly from the works of Cuntz and Power. In Section 3 , we give a general criterion of invertibility of localized endomorphisms. We also present a criterion for a localized endomorphism to restrict to an automorphism of the diagonal. In Section 4 , we develop a labeled tree approach to the search for permutation related automorphisms. We also discuss the effect of inner automorphisms and thus show that unlabeled trees are inner equivalence invariants. In Section 5 we apply the above-mentioned techniques to the case of $\mathcal{O}_{2}$. In Section 6, we give tables summarizing the results of our automorphism search for small values of the parameters $n$ and $k$. These tables were produced through massive computer calculations involving all of the techniques developed in the present paper.

\section{SETup}

If $n$ is an integer greater than 1 , then the Cuntz algebra $\mathcal{O}_{n}$ is a unital, simple $C^{*}$-algebra generated by $n$ isometries $S_{1}, \ldots, S_{n}$, satisfying $\sum_{i=1}^{n} S_{i} S_{i}^{*}=I[\underline{5}$. We denote by $W_{n}^{k}$ the set of $k$-tuples $\alpha=\left(\alpha_{1}, \ldots, \alpha_{k}\right)$ with $\alpha_{m} \in\{1, \ldots, n\}$, and by $W_{n}$ the union $\bigcup_{k=0}^{\infty} W_{n}^{k}$, where $W_{n}^{0}=\{0\}$. We call elements of $W_{n}$ multi-indices. If $\alpha=\left(\alpha_{1}, \ldots, \alpha_{k}\right) \in W_{n}$, then $S_{\alpha}=S_{\alpha_{1}} \ldots S_{\alpha_{k}}$ ( $S_{0}=I$ by convention). Every word in $\left\{S_{i}, S_{i}^{*} \mid i=1, \ldots, n\right\}$ can be uniquely expressed as $S_{\alpha} S_{\beta}^{*}$, for $\alpha, \beta \in W_{n}$ [5. Lemma 1.3]. If $\alpha \in W_{n}^{k}$, then $l(\alpha)=k$, the length of $\alpha$.

$\mathcal{F}_{n}^{k}$ is the $C^{*}$-algebra generated by all words of the form $S_{\alpha} S_{\beta}^{*}, \alpha, \beta \in W_{n}^{k}$, and it is isomorphic to the matrix algebra $M_{n^{k}}(\mathbb{C}) . \mathcal{F}_{n}$, the norm closure of $\bigcup_{k=0}^{\infty} \mathcal{F}_{n}^{k}$, 
is the UHF-algebra of type $n^{\infty}$, called the core UHF-subalgebra of $\mathcal{O}_{n}$ [5. There exists a faithful conditional expectation $F_{0}: \mathcal{O}_{n} \rightarrow \mathcal{F}_{n}[5$.

$\mathcal{D}_{n}$ denotes the diagonal subalgebra of $\mathcal{O}_{n}$, i.e. the $C^{*}$-subalgebra generated by the projections $P_{\alpha}=S_{\alpha} S_{\alpha}^{*}, \alpha \in W_{n}$. As remarked by Cuntz [6, 7], $\mathcal{D}_{n}$ is a maximal abelian subalgebra, regular both in $\mathcal{F}_{n}$ and $\mathcal{O}_{n}$. $\mathcal{D}_{n}$ is naturally isomorphic to $\mathbb{C}\left(X_{n}\right)$, where the spectrum $X_{n}$ is the collection of infinite words in the alphabet $\{1, \ldots, n\}$ [7]. $X_{n}$ with the product topology is a Cantor set, i.e. a compact, metrizable, totally disconnected space with no isolated points. There exists a faithful conditional expectation from $\mathcal{F}_{n}$ onto $\mathcal{D}_{n}$ and whence from $\mathcal{O}_{n}$ onto $\mathcal{D}_{n}$ as well. We denote $\mathcal{D}_{n}^{k}=\mathcal{D}_{n} \cap \mathcal{F}_{n}^{k}$.

Let $P_{n}^{k}$ denote the group of permutations of $W_{n}^{k}$. For $\sigma \in P_{n}^{k}$ there is a corresponding unitary $u \in \mathcal{F}_{n}^{k}$ (we write $u \sim \sigma$ ), $u=\sum_{\alpha \in W_{n}^{k}} S_{\sigma(\alpha)} S_{\alpha}^{*}$. We denote $\mathcal{P}_{n}^{k}=\left\{u \mid \exists \sigma \in P_{n}^{k}, u \sim \sigma\right\}$ and $\mathcal{P}_{n}=\bigcup_{k=0}^{\infty} \mathcal{P}_{n}^{k}$. We have $\mathcal{N}_{\mathcal{F}_{n}}\left(\mathcal{D}_{n}\right)=\mathcal{U}\left(\mathcal{D}_{n}\right) \cdot \mathcal{P}_{n}$, where $\mathcal{N}_{\mathcal{F}_{n}}\left(\mathcal{D}_{n}\right)$ denotes the (unitary) normalizer of $\mathcal{D}_{n}$ in $\mathcal{F}_{n}$ and $\mathcal{U}\left(\mathcal{D}_{n}\right)$ is the unitary group of $\mathcal{D}_{n}$ (see the paragraph preceding Theorem 2.1).

For $B \subseteq A$ algebras, we denote $\operatorname{Aut}(A, B)=\{\sigma \in \operatorname{Aut}(A) \mid \sigma(B)=B\}$, $\operatorname{Aut}_{B}(A)=\left\{\sigma \in \operatorname{Aut}(A)|\sigma|_{B}=\mathrm{id}_{B}\right\}, \operatorname{Inn}(A)$ the inner automorphisms, $\operatorname{Out}(A)=$ $\operatorname{Aut}(A) / \operatorname{Inn}(A)$, and $\pi: \operatorname{Aut}(A) \rightarrow \operatorname{Out}(A)$ the canonical quotient map.

We recall some notation and results from [6]. $\operatorname{End}\left(\mathcal{O}_{n}\right)$ is a semigroup (with composition) of unital endomorphisms of $\mathcal{O}_{n}$. We have a canonical map $\varphi \in$ $\operatorname{End}\left(\mathcal{O}_{n}\right), \varphi(a)=\sum_{i=1}^{n} S_{i} a S_{i}^{*}$. There is a map $\lambda: \mathcal{U}\left(\mathcal{O}_{n}\right) \rightarrow \operatorname{End}\left(\mathcal{O}_{n}\right)$, determined by $\lambda_{u}\left(S_{i}\right)=u^{*} S_{i}$. $\quad \lambda$ is a semigroup isomorphism if $\mathcal{U}\left(\mathcal{O}_{n}\right)$ is equipped with the convolution multiplication $u * w=u \lambda_{u}(w)$. The inverse of $\lambda$ is the map $\rho \mapsto \sum_{i=1}^{n} S_{i} \rho\left(S_{i}^{*}\right)$. Furthermore, $\left.\operatorname{Aut}\left(\mathcal{O}_{n}\right)=\left\{\lambda_{u} \mid u^{*} \in \lambda_{u}\left(\mathcal{O}_{n}\right)\right\}\right\}^{1}$ and $\operatorname{Inn}\left(\mathcal{O}_{n}\right)=\left\{\lambda_{u} \mid u=\varphi(w) w^{*}, w \in \mathcal{U}\left(\mathcal{O}_{n}\right)\right\}$. The map $\mathcal{U}\left(\mathcal{O}_{n}\right) / \mathbb{T} 1 \rightarrow \operatorname{Inn}\left(\mathcal{O}_{n}\right)$, given by $u \mapsto \lambda_{\varphi(u) u^{*}}=\operatorname{Ad}(u)$, is a group isomorphism. We say that $\lambda_{u}$ is invertible if $\lambda_{u} \in \operatorname{Aut}\left(\mathcal{O}_{n}\right)$. For $E \subseteq \mathcal{U}\left(\mathcal{O}_{n}\right)$ we denote $\lambda(E)^{-1}=\left\{\lambda_{u} \mid u \in E\right\} \cap \operatorname{Aut}\left(\mathcal{O}_{n}\right)$.

As shown in [6] we have $\operatorname{Aut}\left(\mathcal{O}_{n}, \mathcal{D}_{n}\right)=\lambda\left(\mathcal{N}_{\mathcal{O}_{n}}\left(\mathcal{D}_{n}\right)\right)^{-1}$ and $\operatorname{Aut}_{\mathcal{D}_{n}}\left(\mathcal{O}_{n}\right)=$ $\lambda\left(\mathcal{U}\left(\mathcal{D}_{n}\right)\right)^{-1} \simeq \mathcal{U}\left(\mathcal{D}_{n}\right)$. More recently, Power determined in [21] (see also [9, 22]) the structure of $\mathcal{N}_{\mathcal{O}_{n}}\left(\mathcal{D}_{n}\right)$. Namely, every $w \in \mathcal{N}_{\mathcal{O}_{n}}\left(\mathcal{D}_{n}\right)$ has a unique decomposition as $w=t u$ with $t \in \mathcal{U}\left(\mathcal{D}_{n}\right)$ and $u$ a finite sum of words. That is, $u$ is a unitary such that $u=\sum_{j=1}^{m} S_{\alpha_{j}} S_{\beta_{j}}^{*}$ for some $\alpha_{j}, \beta_{j} \in W_{n}$. Clearly, such unitaries form a group, which we denote by $\mathcal{S}_{n}$, and this group acts on $\mathcal{U}\left(\mathcal{D}_{n}\right)$ by conjugation. Thus, Power's result says that $\mathcal{N}_{\mathcal{O}_{n}}\left(\mathcal{D}_{n}\right)$ has the structure of a semi-direct product $\mathcal{U}\left(\mathcal{D}_{n}\right) \rtimes \mathcal{S}_{n}$. Therefore, one obtains the following result [26, 18].

Theorem 2.1. $\operatorname{Aut}\left(\mathcal{O}_{n}, \mathcal{D}_{n}\right) \simeq \mathcal{U}\left(\mathcal{D}_{n}\right) \rtimes \lambda\left(\mathcal{S}_{n}\right)^{-1}$. In particular, $\lambda\left(\mathcal{S}_{n}\right)^{-1}$ is a subgroup of $\operatorname{Aut}\left(\mathcal{O}_{n}, \mathcal{D}_{n}\right)$.

Turning back to automorphisms which preserve both the diagonal and the UHFsubalgebra, one easily deduces from the above that $\mathcal{N}_{\mathcal{F}_{n}}\left(\mathcal{D}_{n}\right)=\mathcal{U}\left(\mathcal{D}_{n}\right) \rtimes \mathcal{P}_{n}$ as $\mathcal{P}_{n}=\mathcal{S}_{n} \cap \mathcal{F}_{n}$. Consequently, one has the following result [26].

Theorem 2.2. $\operatorname{Aut}\left(\mathcal{O}_{n}, \mathcal{D}_{n}\right) \cap \operatorname{Aut}\left(\mathcal{O}_{n}, \mathcal{F}_{n}\right)=\lambda\left(\mathcal{N}_{\mathcal{F}_{n}}\left(\mathcal{D}_{n}\right)\right)^{-1} \simeq \lambda\left(\mathcal{U}\left(\mathcal{D}_{n}\right)\right) \rtimes$ $\lambda\left(\mathcal{P}_{n}\right)^{-1}$. In particular, $\lambda\left(\mathcal{P}_{n}\right)^{-1}$ is a subgroup of $\operatorname{Aut}\left(\mathcal{O}_{n}, \mathcal{D}_{n}\right) \cap \operatorname{Aut}\left(\mathcal{O}_{n}, \mathcal{F}_{n}\right)$.

Proof. At first we show that $\operatorname{Aut}\left(\mathcal{O}_{n}, \mathcal{D}_{n}\right) \cap \operatorname{Aut}\left(\mathcal{O}_{n}, \mathcal{F}_{n}\right)=\lambda\left(\mathcal{N}_{\mathcal{F}_{n}}\left(\mathcal{D}_{n}\right)\right)^{-1}$. If $\lambda_{w} \in$ $\operatorname{Aut}\left(\mathcal{O}_{n}, \mathcal{D}_{n}\right) \cap \operatorname{Aut}\left(\mathcal{O}_{n}, \mathcal{F}_{n}\right)$, then it follows from [6, Prop. 1.5, Prop. 1.2(b)] that $w \in \mathcal{N}_{\mathcal{O}_{n}}\left(\mathcal{D}_{n}\right) \cap \mathcal{F}_{n}=\mathcal{N}_{\mathcal{F}_{n}}\left(\mathcal{D}_{n}\right)$. On the other hand, if $w \in \mathcal{N}_{\mathcal{F}_{n}}\left(\mathcal{D}_{n}\right)$ and

\footnotetext{
${ }^{1}$ In general, it may happen that $\lambda_{u}$ is an automorphism but $\lambda_{u^{*}}$ is not.
} 
$\lambda_{w} \in \operatorname{Aut}\left(\mathcal{O}_{n}\right)$, then $\lambda_{w} \in \operatorname{Aut}\left(\mathcal{O}_{n}, \mathcal{D}_{n}\right)$ and $\lambda_{w}\left(\mathcal{F}_{n}\right) \subset \mathcal{F}_{n}$ and the conclusion follows immediately from [26, Lemma 2].

Let $u \in \mathcal{P}_{n}$ and let $\lambda_{u}$ be invertible. Then $\lambda_{u}^{-1}$ belongs to $\operatorname{Aut}\left(\mathcal{O}_{n}, \mathcal{D}_{n}\right) \cap$ $\operatorname{Aut}\left(\mathcal{O}_{n}, \mathcal{F}_{n}\right)$ and thus $\lambda_{u}^{-1}=\lambda_{z}$ with $z \in \mathcal{N}_{\mathcal{F}_{n}}\left(\mathcal{D}_{n}\right)$. Thus, by 21, Lemma 5.4, (i)], there are $v \in \mathcal{P}_{n}$ and $y \in \mathcal{U}\left(\mathcal{D}_{n}\right)$ such that $z=v y$. We have id $=\lambda_{u} \lambda_{v y}$ and hence $1=u \lambda_{u}(v) \lambda_{u}(y)$. Thus $\mathcal{P}_{n} \ni u \lambda_{u}(v)=\lambda_{u}\left(y^{*}\right) \in \mathcal{U}\left(\mathcal{D}_{n}\right)$. Therefore $y=1$ and consequently $\lambda_{u}^{-1}=\lambda_{v}$. It follows that $\lambda\left(\mathcal{P}_{n}\right)^{-1}$ is a subgroup of $\operatorname{Aut}\left(\mathcal{O}_{n}, \mathcal{D}_{n}\right) \cap \operatorname{Aut}\left(\mathcal{O}_{n}, \mathcal{F}_{n}\right)$. Clearly, $\lambda\left(\mathcal{P}_{n}\right)^{-1}$ acts on $\operatorname{Aut}_{\mathcal{D}_{n}}\left(\mathcal{O}_{n}\right)=\lambda\left(\mathcal{U}\left(\mathcal{D}_{n}\right)\right)$ by conjugation.

Now, by Theorem 2.1, $\lambda_{w} \in \operatorname{Aut}\left(\mathcal{O}_{n}, \mathcal{D}_{n}\right) \cap \operatorname{Aut}\left(\mathcal{O}_{n}, \mathcal{F}_{n}\right)$ can be uniquely written as a product of two elements from $\lambda\left(\mathcal{S}_{n}\right)^{-1}$ and $\lambda\left(\mathcal{U}\left(\mathcal{D}_{n}\right)\right), \lambda_{w}=\lambda_{u} \lambda_{s^{*}}, u \in \mathcal{S}_{n}$, $s \in \mathcal{D}_{n}$. But then $\lambda_{w} \lambda_{s}\left(\mathcal{F}_{n}\right)=\mathcal{F}_{n}$ and $u \in \mathcal{S}_{n} \cap \mathcal{F}_{n}=\mathcal{P}_{n}$.

A slightly weaker version of the following lemma was given in [26].

Lemma 2.3. Let $w \in \mathcal{P}_{n}$. If $\lambda_{w} \in \operatorname{Inn}\left(\mathcal{O}_{n}\right)$, then there exists $u \in \mathcal{P}_{n}$ such that $w=\varphi(u) u^{*}$. Moreover, for $k \geq 2$, if $w \in \mathcal{P}_{n}^{k}$, then $u \in \mathcal{P}_{n}^{k-1}$.

Proof. The proof of the first statement can be found in [26]. Suppose that $w=$ $\varphi(u) u^{*} \in \mathcal{F}_{n}^{k}$ with $u \in \mathcal{F}_{n}^{h}$ for some $h$. Observe that if $h \geq k$, then $\mathcal{F}_{n}^{h} \supset \mathcal{F}_{n}^{k}$ so that $\varphi(u) \in \mathcal{F}_{n}^{h}$ and $u \in \mathcal{F}_{n}^{h-1}$. Therefore $h<k$ and necessarily one must have $h=k-1$.

Since $\mathcal{P}_{n} \simeq \lambda\left(\mathcal{P}_{n}\right)^{-1} \cap \operatorname{Inn}\left(\mathcal{O}_{n}\right)$ via $u \mapsto \operatorname{Ad}(u)$ [6], there exists an exact sequence

$$
1 \rightarrow \mathcal{P}_{n} \rightarrow \lambda\left(\mathcal{P}_{n}\right)^{-1} \rightarrow \pi\left(\lambda\left(\mathcal{P}_{n}\right)^{-1}\right) \rightarrow 1
$$

The natural inclusion $\mathcal{P}_{n}^{k} \subset \mathcal{P}_{n}^{k+m}$ corresponds to the embedding $P_{n}^{k} \hookrightarrow P_{n}^{k+m}$, $\phi \mapsto \phi \times \mathrm{id}_{m}$, where $\mathrm{id}_{m}$ denotes the identity on $W_{n}^{m}$ (we have $W_{n}^{k+m}=W_{n}^{k} \times W_{n}^{m}$ ). With this identification $P_{n}=\bigcup_{k} P_{n}^{k}$ becomes a group isomorphic to $\mathcal{P}_{n}$. We note that the imbedding $\mathcal{P}_{n}^{k} \hookrightarrow \mathcal{P}_{n}^{k+m}, u \mapsto \varphi^{m}(u)$, corresponds to the imbedding $P_{n}^{k} \hookrightarrow P_{n}^{k+m}, \phi \mapsto \operatorname{id}_{m} \times \phi$. If $\phi \in P_{n}^{k}$ and $r \geq 1$, then we define $\phi^{(r)} \in P_{n}^{k+r-1}$ as

$$
\phi^{(r)}=\left(i d_{r-1} \times \phi\right)\left(\operatorname{id}_{r-2} \times \phi \times \operatorname{id}_{1}\right) \ldots\left(\phi \times \operatorname{id}_{r-1}\right) .
$$

In particular, $\phi^{(1)}=\phi$. For $u \in \mathcal{P}_{n}^{k}, u \sim \phi, w=\varphi(u) u^{*}$ (i.e., $\lambda_{w}=\operatorname{Ad}(u)$ ), $w \sim \psi$, we have $\psi=\left(\operatorname{id}_{1} \times \phi\right)\left(\phi^{-1} \times \operatorname{id}_{1}\right), \psi^{(r)}=\left(\operatorname{id}_{r} \times \phi\right)\left(\phi^{-1} \times \operatorname{id}_{r}\right)$ and thus $\psi^{(k)}=\phi^{-1} \times \phi$.

The map $\mathcal{P}_{n}^{k} \times \mathcal{P}_{n}^{r} \rightarrow \mathcal{P}_{n}^{k+r-1},(u, w) \mapsto u * w=u \lambda_{u}(w)$ corresponds to the map $P_{n}^{k} \times P_{n}^{r} \rightarrow P_{n}^{k+r-1}$,

$$
(\alpha, \beta) \mapsto \alpha * \beta=\left(\alpha \times \operatorname{id}_{r-1}\right)\left(\alpha^{(r)}\right)^{-1}\left(\beta \times \operatorname{id}_{k-1}\right) \alpha^{(r)} .
$$

If a permutation $\phi \in P$ is $*$-invertible, then we denote its inverse by $\bar{\phi}$.

For later use we highlight a simple but suggestive reformulation of the second statement in Theorem 2.2.

Proposition 2.4. Let $w \in \mathcal{P}_{n}^{k}$ and suppose that $\lambda_{w} \in \operatorname{Aut}\left(\mathcal{O}_{n}\right)$. Then $\lambda_{w}^{-1}$ is also induced by a unitary in $\mathcal{P}_{n}^{h}$, for some $h$.

We will see later that indeed $h \leq n^{2(k-1)}$; see Corollary 3.3 .

Following 4, we call "localized" an endomorphism of the Cuntz algebra $\mathcal{O}_{n}$ of the form $\lambda_{u}$ with $u$ a unitary in $\bigcup_{k} \mathcal{F}_{n}^{k}$. Of course, by the very definition, all the $\lambda_{w}$ 's with $w \in \mathcal{P}_{n}^{k}$ are localized endomorphisms and, by the above, automorphisms 
induced by permutation unitaries are examples of localized automorphisms whose inverse is (induced by a permutation unitary and thus) still localized.

Now the natural question arises whether one can find an effective algorithm to identify all the permutation unitaries inducing automorphisms of the Cuntz algebra. Eventually, one might also like to give a closer look at the properties of these (possibly outer) automorphisms. Especially, one interesting problem is to determine the structure of the groups $\pi\left(\lambda\left(\mathcal{P}_{n}\right)^{-1}\right)$ for $n \geq 2$. As shown in 26, Example 9], the groups $\pi\left(\lambda\left(\mathcal{P}_{n}\right)^{-1}\right)$ for $n \geq 3$ are quite "big" in the sense that they contain nonamenable subgroups, notably $\mathbb{Z}_{2} * \mathbb{Z}_{3}$. The same question for $n=2$ is more subtle. On the basis of general results [24, 11, 20, it has been known for some time that the automorphism group of $\mathcal{O}_{2}$ is in some sense considerably "smaller". Our computations provide very concrete evidence to this effect.

\section{Searching For automorphisms}

3.1. Invertibility of localized endomorphisms. In this subsection, we obtain the crucial Theorem 3.2 as the result of a clarifying interaction between the ideas in [26] and in [4. We start recalling the argument in [26, Theorem 7] and then explain how to combine it with the analysis in [4, notably Proposition 6.1 therein, to get indeed a very satisfactory picture that opens the way to the combinatorial analysis discussed in the next section.

Let $w \in \mathcal{P}_{n}^{k}$ be a permutation unitary. We set

$$
B_{w}=\left\{w, \varphi(w), \ldots, \varphi^{k-2}(w)\right\}^{\prime} \cap \mathcal{F}_{n}^{k-1}
$$

if $k \geq 2$ and $B_{w}=\mathbb{C} 1$ otherwise. That is, $b \in \mathcal{F}_{n}^{k-1}$ is in $B_{w}$ if and only if, for any $\alpha, \beta \in W_{n}^{l}, l \in\{0, \ldots, k-2\}, S_{\alpha}^{*} b S_{\beta}$ commutes with $w$. Of course, $B_{w}\left(=B_{w^{*}}\right)$ is a unital $*$-subalgebra of $\mathcal{F}_{n}^{k-1}$. Notice that if $b \in B_{w}$, then $\lambda_{w}(b)=b$. Also, for $i, j \in\{1, \ldots, n\}$ we define maps $a_{i j}^{w}: \mathcal{F}_{n}^{k-1} \rightarrow \mathcal{F}_{n}^{k-1}$ by

$$
a_{i j}^{w}(x)=S_{i}^{*} w x w^{*} S_{j}, \quad x \in \mathcal{F}_{n}^{k-1} .
$$

We denote $V_{w}=\mathcal{F}_{n}^{k-1} / B_{w}$. Since $a_{i j}^{w}\left(B_{w}\right) \subseteq B_{w}$, there are induced maps $\tilde{a}_{i j}^{w}$ : $V_{w} \rightarrow V_{w}$. We define $A_{w}$ as the subring of $\mathcal{L}\left(V_{w}\right)$ generated by $\left\{\tilde{a}_{i j}^{w} \mid i, j=1, \ldots, n\right\}$.

Lemma 3.1 ([26]). If $w \in \mathcal{P}_{n}$, then $\lambda_{w}$ is invertible if and only if $A_{w}$ is nilpotent.

Proof. Necessity. Let $w \in \mathcal{P}_{n}^{k}$ and $\lambda_{w}$ be invertible. By Proposition 2.4, $\lambda_{w}^{-1}$ is then induced by some (permutation) unitary in some finite matrix algebra. Let $\lambda_{w}^{-1}\left(\mathcal{F}_{n}^{k-1}\right) \subseteq \mathcal{F}_{n}^{l}$. For $a \in \mathcal{F}_{n}^{l}$ the sequence $\operatorname{Ad}\left(w^{*} \varphi\left(w^{*}\right) \ldots \varphi^{m}\left(w^{*}\right)\right)(a)$ stabilizes from $m=l-1$ at $\lambda_{w}(a)$. Consequently, for any $b \in \mathcal{F}_{n}^{k-1}$ the sequence $\operatorname{Ad}\left(\varphi^{m}(v) \ldots \varphi(w) w\right)(b)$ stabilizes from $m=l-1$ at $\lambda_{w}^{-1}(b)$. There are $c_{\gamma \rho} \in \mathbb{C} 1$ such that

$$
\sum_{\gamma, \rho \in W_{n}^{l}} S_{\gamma} c_{\gamma \rho}(b) S_{\rho}^{*}=\operatorname{Ad}\left(\varphi^{l-1}(w) \ldots \varphi(w) w\right)(b) \in \mathcal{F}_{n}^{l} .
$$

If $\alpha=\left(i_{1}, \ldots, i_{l}\right), \beta=\left(j_{1}, \ldots, j_{l}\right), T_{\alpha, \beta}=a_{i_{l} j_{l}}^{w} \ldots a_{i_{1} j_{1}}^{w}$, and $b \in \mathcal{F}_{n}^{k-1}$, then $T_{\alpha, \beta}(b)=c_{\alpha \beta}(b) \in \mathbb{C} 1 \subset B_{w}$. Consequently, $A_{w}^{l}=0$.

Sufficiency. Let $w \in \mathcal{P}_{n}^{k}$ and assume that $A_{w}^{l}=0$. Let $b \in \mathcal{F}_{n}^{k-1}$ and let $T_{\alpha, \beta}$ be as above. By hypothesis, $T_{\alpha, \beta}(b)$ commutes with $\varphi^{m}(w)$ for any $m$. Hence, if 
$r \geq 1$, we have

$$
\begin{aligned}
\operatorname{Ad}\left(\varphi^{l-1+r}(w) \ldots \varphi(w) w\right)(b) & =\operatorname{Ad}\left(\varphi^{l-1+r}(w) \ldots \varphi^{l}(w)\right)\left(\sum_{\alpha, \beta \in W_{n}^{l}} S_{\alpha} T_{\alpha, \beta}(b) S_{\beta}^{*}\right) \\
& =\sum_{\alpha, \beta \in W_{n}^{l}} S_{\alpha} \operatorname{Ad}\left(\varphi^{r-1}(w) \ldots w\right)\left(T_{\alpha, \beta}(b)\right) S_{\beta}^{*} \\
& =\sum_{\alpha, \beta \in W_{n}^{l}} S_{\alpha} T_{\alpha, \beta}(b) S_{\beta}^{*} .
\end{aligned}
$$

Thus, for any $b \in \mathcal{F}_{n}^{k-1}$, the sequence $\operatorname{Ad}\left(\varphi^{m}(w) \ldots \varphi(w) w\right)(b)$ stabilizes from $m=$ $l-1$. Let $w^{*}=\sum_{i, j=1}^{n} S_{i} b_{i j} S_{j}^{*}, b_{i j} \in \mathcal{F}_{n}^{k-1}$. By the above, the sequence

$$
\begin{aligned}
\operatorname{Ad}\left(\varphi^{m}(w) \ldots \varphi(w) w\right)\left(w^{*}\right) & =\sum_{i, j=1}^{n} \operatorname{Ad}\left(\varphi\left(\varphi^{m-1} \ldots \varphi(w) w\right)\right)\left(S_{i} b_{i j} S_{j}^{*}\right) \\
& =\sum_{i, j} S_{i} \operatorname{Ad}\left(\varphi^{m-1}(w) \ldots \varphi(w) w\right)\left(b_{i j}\right) S_{j}^{*}
\end{aligned}
$$

stabilizes from $m=l$ at $\lambda_{w}^{-1}\left(w^{*}\right)$ and hence $\lambda_{w}$ is invertible.

In turn, inspection of the proof shows that a similar characterization holds true for any unitary $u \in \mathcal{F}_{n}^{k}$ such that $\lambda_{u}$ is invertible with a localized inverse. (If $\lambda_{u}^{-1}=\lambda_{v}$ with $v \in \mathcal{F}_{n}^{h}$, one can choose $l=k+h-2$ in the above argument.) Moreover, we can adapt some arguments from section 6 of 4 to our situation. We denote by $H$ the linear span of the $S_{i}$ 's. Given a unitary $u \in \mathcal{F}_{n}^{k}$, following [4, p. 386], we define inductively

$$
\Xi_{0}=\mathcal{F}_{n}^{k-1}, \quad \Xi_{r}=\lambda_{u}(H)^{*} \Xi_{r-1} \lambda_{u}(H), r \geq 1
$$

that is, $\Xi_{r}=\left(\lambda_{u}(H)^{r}\right)^{*} \mathcal{F}_{n}^{k-1}\left(\lambda_{u}(H)\right)^{r}$. It readily follows that $\left(\Xi_{r}\right)_{r}$ is a nonincreasing sequence of subspaces of $\mathcal{F}_{n}^{k-1}$ that stabilizes at the first value $p$ for which $\Xi_{p}=\Xi_{p+1}$. Let $\Xi_{u}:=\bigcap_{r} \Xi_{r}=\Xi_{p}$.

Theorem 3.2. Let $u$ be a unitary in $\mathcal{F}_{n}^{k}$ for some $k \geq 1$. Then the following conditions are equivalent:

(1) $\lambda_{u}$ is invertible with localized inverse;

(2) the sequence of unitaries

$$
\left(\operatorname{Ad}\left(\varphi^{m}(u) \varphi^{m-1}(u) \ldots \varphi(u) u\right)\left(u^{*}\right)\right)_{m \geq 1}
$$

eventually stabilizes;

(3) $A_{u}$ is nilpotent;

(4) $\Xi_{u} \subseteq B_{u}$;

(5) $\Xi_{u}=\mathbb{C} 1$.

Proof. (1) $\Rightarrow(2)$ : Let $v \in \mathcal{F}_{n}^{h}$ be such that $\lambda_{u} \lambda_{v}=$ id. Thus $u \lambda_{u}(v)=1$; that is,

$$
u^{*} \varphi\left(u^{*}\right) \cdots \varphi^{m}\left(u^{*}\right) v \varphi^{m}(u) \cdots \varphi(u) u=u^{*}
$$

for every $m \geq h-1$.

$(2) \Rightarrow(1)$ : Suppose that there exists some positive integer $l$ for which

$$
\varphi^{m}(u) \ldots \varphi(u) u^{*} \varphi\left(u^{*}\right) \ldots \varphi^{m}\left(u^{*}\right)=\varphi^{l}(u) \ldots \varphi(u) u^{*} \varphi\left(u^{*}\right) \ldots \varphi^{l}\left(u^{*}\right)
$$

for every $m \geq l$. Call $v$ the resulting unitary, clearly in $\mathcal{F}_{n}^{k+l}$. Then $u \lambda_{u}(v)=$ $u\left(u^{*} \ldots \varphi^{k+l-1}\left(u^{*}\right)\right) v\left(\varphi^{k+l-1}(u) \ldots u\right)=u u^{*}=1$, and therefore $\lambda_{v}=\lambda_{u}^{-1}$. 
The equivalence of (1) and (3) follows by Lemma 3.1, mutatis mutandis. As (4) is nothing but a reformulation of the nilpotency condition, (3) and (4) are clearly equivalent.

$(1) \Rightarrow(5)$ : It follows from [4, Proposition 6.1], where we take as $\Phi$ the (normal extension of the) localized automorphism $\lambda_{u}{ }^{-1} 2$

$(5) \Rightarrow(4)$ : This is obvious.

Corollary 3.3. Let $u \in \mathcal{F}_{n}^{k}$ be a unitary satisfying the equivalent conditions of Theorem 3.2. Then $\lambda_{u}^{-1}$ is induced by a unitary $v \in \mathcal{F}_{n}^{h}$ with $h=n^{2(k-1)}$.

Proof. Let $K=\lambda_{u}(H)$. As the sequence of finite dimensional subspaces $\mathcal{F}_{n}^{k-1} \supset$ $K^{*} \mathcal{F}_{n}^{k-1} K \supset K^{* 2} \mathcal{F}_{n}^{k-1} K^{2} \supset \ldots$ is decreasing until it stabilizes to $\mathbb{C}, \operatorname{dim}\left(\mathcal{F}_{n}^{k-1}\right)=$ $n^{2(k-1)}$ and at each step the dimension drops by one at least, one has $\left(K^{*}\right)^{p} \mathcal{F}_{n}^{k-1} K^{p}$ $=\mathbb{C}$ for some $p \leq n^{2(k-1)}-1$.

Next observe that

$$
K^{* p+1} \mathcal{F}_{n}^{k} K^{p+1}=K^{* p} \mathcal{F}_{n}^{k-1} K^{p}=\mathbb{C} .
$$

That is,

$$
H^{* p+1} \varphi^{p}(u) \cdots u \mathcal{F}_{n}^{k} u^{*} \cdots \varphi^{p}\left(u^{*}\right) H^{p+1}=\mathbb{C}
$$

and $v:=\varphi^{p}(u) \cdots u u^{*} u^{*} \cdots \varphi^{p}\left(u^{*}\right) \in \mathcal{F}_{n}^{p+1}$. This shows the statement.

At this stage it is not clear whether it is possible to improve the exponential bound on $h$ in the last corollary. This would be rather useful for computational purposes.

3.2. Automorphisms of the diagonal. It follows from [6, Proposition 1.5] that if $w \in \mathcal{F}_{n}^{k}$ is in the normalizer of the diagonal subalgebra $\mathcal{D}_{n}$, then for $\lambda_{w}$ to be invertible it is necessary that $\lambda_{w}\left(\mathcal{D}_{n}\right)=\mathcal{D}_{n}$. It turns out that the method of Lemma 3.1 and Theorem 3.2 can also provide a criterion of invertibility of the restriction of such an endomorphism $\lambda_{w}$ to the diagonal $\mathcal{D}_{n}$.

Indeed, let $w \in \mathcal{F}_{n}^{k} \cap \mathcal{N}_{\mathcal{O}_{n}}\left(\mathcal{D}_{n}\right)$. Then both $\mathcal{D}_{n}^{k-1}$ and $B_{w} \cap \mathcal{D}_{n}^{k-1}$ are invariant subspaces for all the operators $a_{i j}^{w}$ associated with $w$. Denote the restriction of $a_{i j}^{w}$ to $\mathcal{D}_{n}^{k-1}$ by $b_{i j}^{w}$. Each $b_{i j}^{w}$ induces a linear transformation $\tilde{b}_{i j}^{w}: V_{w}^{D} \rightarrow V_{w}^{D}$, where $V_{w}^{D}=\mathcal{D}_{n}^{k-1} /\left(B_{w} \cap \mathcal{D}_{n}^{k-1}\right)$. We denote by $A_{w}^{D}$ the subring of $\mathcal{L}\left(V_{w}^{D}\right)$ generated by $\left\{\tilde{b}_{i j}^{w} \mid i, j=1, \ldots, n\right\}$. Also, we consider the subspace of $\mathcal{D}_{n}^{k-1}$ defined by $\Xi_{w}^{D}:=\bigcap_{r}\left(K^{*}\right)^{r} \mathcal{D}_{n}^{k-1} K^{r}$, where $K$ is the linear span of $w^{*} S_{1}, \ldots, w^{*} S_{n}$.

Theorem 3.4. Let $w$ be a unitary in $\mathcal{F}_{n}^{k} \cap \mathcal{N}_{\mathcal{O}_{n}}\left(\mathcal{D}_{n}\right)$. If the ring $A_{w}^{D}$ is nilpotent, then $\lambda_{w}$ restricts to an automorphism of $\mathcal{D}_{n}$. More precisely, the following conditions are equivalent:

(1) $\lambda_{w}$ restricts to an automorphism of the algebraic part $\bigcup_{s} \mathcal{D}_{n}^{s}$ of $\mathcal{D}_{n}$;

(2) the ring $A_{w}^{D}$ is nilpotent;

(3) $\Xi_{w}^{D} \subseteq B_{w} \cap \mathcal{D}$;

(4) $\Xi_{w}^{D}=\mathbb{C} 1$.

Proof. We only give details of the proof of the implication $(2) \Rightarrow(1)$. The other implications are established through arguments very similar to those of Lemma 3.1 and Theorem 3.2 .

\footnotetext{
${ }^{2}$ We warn the reader about a slightly confusing change in the conventions. The $\lambda_{u}$ in [4] corresponds to $\lambda_{u^{*}}$ here.
} 
Suppose that $A_{w}^{D}$ is nilpotent. We show by induction on $r \geq k$ that all $\mathcal{D}_{n}^{r}$ are in the range of $\lambda_{w}$ restricted to $\bigcup_{s} \mathcal{D}_{n}^{s}$.

If $x \in \mathcal{D}_{n}^{k}$, then the same argument as in the proof of the sufficiency part in Lemma 3.1 shows that $x$ belongs to $\lambda_{w}\left(\bigcup_{s} \mathcal{D}_{n}^{s}\right)$. In fact, the sequence $\operatorname{Ad}\left(\varphi^{m}(w) \ldots \varphi(w) w\right)(x)$ stabilizes at $\lambda_{w}^{-1}(x) \in \bigcup_{s} \mathcal{D}_{n}^{s}$.

For the inductive step, suppose that $r \geq k$ and $\mathcal{D}_{n}^{r} \subset \lambda_{w}\left(\bigcup_{s} \mathcal{D}_{n}^{s}\right)$. Since $\mathcal{D}_{n}^{r+1}$ is generated by $\mathcal{D}_{n}^{r}$ and $\varphi^{r}\left(\mathcal{D}_{n}^{1}\right)$, it suffices to show that $\varphi^{r}(y)$ belongs to $\lambda_{w}\left(\bigcup_{s} \mathcal{D}_{n}^{s}\right)$ for all $y \in \mathcal{D}_{n}^{1}$. However, $\varphi^{r}(y)$ commutes with $w$ and $\varphi^{r-1}(y) \in \mathcal{D}_{n}^{r}$ is in $\lambda_{w}\left(\bigcup_{s} \mathcal{D}_{n}^{s}\right)$. Thus, we see that the sequence

$$
\operatorname{Ad}\left(\varphi^{m}(w) \ldots \varphi(w) w\right)\left(\varphi^{r}(y)\right)=\varphi\left(\operatorname{Ad}\left(\varphi^{m-1}(w) \ldots \varphi(w) w\right)\left(\varphi^{r-1}(y)\right)\right)
$$

stabilizes at $\lambda_{w}^{-1}\left(\varphi^{r}(y)\right) \in \bigcup_{s} \mathcal{D}_{n}^{s}$.

It is not difficult to verify that if $w \in \mathcal{P}_{n}$ and $\lambda_{w}\left(\mathcal{D}_{n}\right)=\mathcal{D}_{n}$, then $\lambda_{w}$ satisfies condition (1) of Theorem 3.4 .

\section{Applications of labeled trees to the search for automorphisms}

Let $w \in \mathcal{P}_{n}^{k}$. Take $\left\{S_{\alpha} S_{\beta}^{*}\right\}_{\alpha, \beta \in W_{n}^{k-1}}$, a basis of $\mathcal{F}_{n}^{k-1}$, so that $\left\{S_{\alpha} S_{\alpha}^{*}\right\}$ are the first block of the basis. With respect to this basis, each $a_{i j}^{w}, i, j \in\{1, \ldots, n\}$ has a matrix

$$
a_{i j}^{w}=\left(\begin{array}{cc}
b_{i j}^{w} & c_{i j}^{w} \\
0 & d_{i j}^{w}
\end{array}\right)
$$

with entries in $\{0,1\}$, as $a_{i j}^{w}\left(S_{\alpha} S_{\beta}^{*}\right)=\sum_{m} S_{i}^{*} S_{\sigma(\alpha, m)} S_{\sigma(\beta, m)}^{*} S_{j}$, where $w \sim \sigma$.

In the sequel of this section, we will explain how the condition that $\lambda_{w} \in \operatorname{Aut}\left(\mathcal{O}_{n}\right)$ for $w \in \mathcal{P}_{n}^{k}$ translates in terms of the $a_{i j}^{w}$ 's. In turn, this boils down to two separate arguments for the (sub)matrices $[b]$ and $[d]$. As a matter of fact, $[c]$ turns out to be irrelevant for the following discussion. Indeed, since $a_{i j}^{w}(I) \in\{I, 0\}$, each $a_{i j}^{w}$ gives rise to a map from $\mathcal{F}_{n}^{k-1} / \mathbb{C}$ to itself, whose matrix has a block form

$$
\left(\begin{array}{cc}
\hat{b}_{i j}^{w} & * \\
0 & d_{i j}^{w}
\end{array}\right)
$$

It is an immediate corollary of Lemma 3.1 and Theorem 3.2 that $\lambda_{w}$ is invertible if and only if both rings generated by $\left\{\hat{b}_{i j}^{w} \mid i, j=1, \ldots, n\right\}$ and by $\left\{d_{i j}^{w} \mid i, j=\right.$ $1, \ldots, n\}$, respectively, are nilpotent. Furthermore, it follows from Theorem 3.4 that nilpotency of the ring generated by $\left\{\hat{b}_{i j}^{w} \mid i, j=1, \ldots, n\right\}$ implies that the endomorphism $\lambda_{w}$ restricts to an automorphism of $\mathcal{D}_{n}$.

4.1. Upper left corner $[b]$. The plan of this subsection is as follows. We first convert the matrix $[b]$ into functions on indices. Trees then pop up as diagrams of these functions. Next we discuss labeling. The automorphism condition will lead us to trees with a suitable labeling, that is, inducing a certain partial order relation.

If $i \neq j$, then $b_{i j}^{w}=0$. Hence we can write $b_{i}^{w}:=b_{i i}^{w}$. Since

$$
b_{i}^{w}\left(S_{\alpha} S_{\alpha}^{*}\right)=\sum_{m} S_{i}^{*} w S_{\alpha} S_{m} S_{m}^{*} S_{\alpha}^{*} w^{*} S_{i}
$$

$w S_{\alpha} S_{m} S_{m}^{*} S_{\alpha}^{*} w^{*}$ being a minimal projection in $\mathcal{D}_{n}^{k}$, it follows that each column of $b_{i}^{w}$ has at most $n$ nonzero entries, but fixing a column and summing over $i$ we get 
exactly $n$. Furthermore, since $b_{i}^{w}(1)=1$, we have

$$
\sum_{\alpha} S_{\alpha} S_{\alpha}^{*}=\sum_{\alpha} b_{i}^{w}\left(S_{\alpha} S_{\alpha}^{*}\right)
$$

and hence each row of $b_{i}^{w}$ has exactly one 1 and the rest 0 .

Suppose that $\lambda_{w}$ is an automorphism of $\mathcal{O}_{n}$. Then the equivalence of conditions (1), (3) and (5) of Theorem 3.2 easily implies the following condition on the upper left corner of the matrix $\left[a_{i j}^{w}\right]$ : sufficiently long products of the operators $\left\{b_{i}^{w} \mid i=\right.$ $1, \ldots, n\}$ have the form

$$
\left(\begin{array}{cccc}
\lambda_{1} & \lambda_{2} & \cdots & \lambda_{n^{k-1}} \\
\lambda_{1} & \lambda_{2} & \cdots & \lambda_{n^{k-1}} \\
\vdots & \vdots & & \vdots \\
\lambda_{1} & \lambda_{2} & \cdots & \lambda_{n^{k-1}}
\end{array}\right) ;
$$

that is, they are constant along the columns. However, since for any $i$ each row of $b_{i}^{w}$ contains exactly one nonzero entry, the same is true for products of the $\left\{b_{i}^{w}\right\}$ s. Thus each of the above matrices as in (11) must actually have the form

$$
\left(\begin{array}{ccccc}
0 & \cdots & 1 & \cdots & 0 \\
0 & \cdots & 1 & \cdots & 0 \\
\vdots & & \vdots & & \vdots \\
0 & \cdots & 1 & \cdots & 0
\end{array}\right)
$$

i.e. one column of 1's and 0's elsewhere.

Since each row of the matrix $b_{i}^{w}$ has a 1 exactly in one column and 0's elsewhere, the $b_{i}^{w}$ can be identified with a function $f_{i}^{w}: W_{n}^{k-1} \rightarrow W_{n}^{k-1}$ defined by

$$
f_{i}^{w}(\alpha)=\beta
$$

whenever $b_{i}^{w}$ has a 1 in the $(\alpha, \beta)$ entry. Suppose that $w$ comes from a permutation $\sigma$. Then

$$
\begin{aligned}
f_{i}^{w}(\alpha)=\beta & \Longleftrightarrow \exists m \text { such that }(i, \alpha)=\sigma(\beta, m) \\
& \Longleftrightarrow S_{\alpha} S_{\alpha}^{*} \leq S_{i}^{*} w S_{\beta} S_{\beta}^{*} w^{*} S_{i} .
\end{aligned}
$$

It is not difficult to verify that the product $b_{i}^{w} b_{j}^{w}$ corresponds to the composition $f_{j}^{w} \circ f_{i}^{w}$ (in reversed order of $i$ and $j$ ). In what follows we often omit the superscript $w$ in $f_{i}^{w}$ when no confusion may arise.

We omit an easy proof of the following lemma.

Lemma 4.1. The ring generated by $\left\{\hat{b}_{i}^{w} \mid i=1, \ldots, n\right\}$ is nilpotent if and only if all sufficiently long composition products of mappings $\left\{f_{i} \mid i=1, \ldots, n\right\}$ have ranges consisting of a single element.

Lemma 4.2. A necessary condition of nilpotency of the ring generated by $\left\{\hat{b}_{i}^{w} \mid i=\right.$ $1, \ldots, n\}$ is that each $f_{i}$ must have the following structure:

- exactly one fixed-point;

- no periodic orbits of length $\geq 2$.

Proof. The first condition clearly follows by considering, for any given index $i$, only powers of the matrix $b_{i}$ or, equivalently, compositions of the same function $f_{i}$. The second condition follows since otherwise some power of $b_{i}$ would have more than one fixed point. 
From this lemma we deduce that the diagrams of the $f_{i}$ 's are rooted trees, where the root corresponds to the unique fixed point. By a diagram we mean the graph with vertices labeled by elements of $W_{n}^{k-1}$ and with a directed edge from vertex $\alpha$ to vertex $\beta$ if $f_{i}(\alpha)=\beta$. By convention, we do not include in the diagram the loop from the root (fixed point) to itself.

Example 4.3. The pair of labeled trees corresponding to $\sigma=$ id in $P_{2}^{3}$. All the edges are downward oriented.
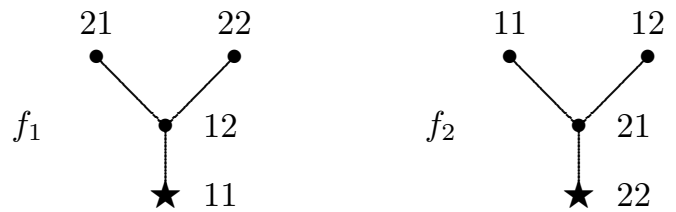

Example 4.4. Let $u \in \mathcal{P}_{n}^{1}$, so that $\lambda_{u}$ is a Bogolubov automorphism of $\mathcal{O}_{n}$. If we view $u$ as an element of $\mathcal{P}_{n}^{k}$, then all $n$ unlabeled trees corresponding to $u$ are identical; the root receives $n-1$ edges from other vertices, each other vertex receives either none or $n$ edges, and the height of the tree (the length of the longest path ending at the root) is minimal and equal to $k-1$. In particular, all such unitaries have the corresponding $n$-tuples of unlabeled trees identical with those of the identity.

Lemma 4.5. The ring generated by $\left\{\hat{b}_{i}^{w} \mid i=1, \ldots, n\right\}$ is nilpotent if and only if there exists a partial order $\leq$ on the cartesian product $W_{n}^{k-1} \times W_{n}^{k-1}$ such that:

(i) Each element of the diagonal $(\alpha, \alpha)$ is minimal.

(ii) Each $(\alpha, \beta)$ is bounded below by some diagonal element.

(iii) For every $i$ and all $(\alpha, \beta)$ such that $\alpha \neq \beta$, we have

$$
\left(f_{i}(\alpha), f_{i}(\beta)\right) \leq(\alpha, \beta) \text {. }
$$

Proof. Suppose that the ring generated by $\left\{\hat{b}_{i}^{w} \mid i=1, \ldots, n\right\}$ is nilpotent. Define a relation $\leq$ as follows. For any $\alpha,(\alpha, \alpha) \leq(\alpha, \alpha)$. If $\gamma \neq \delta$, then $(\alpha, \beta) \leq(\gamma, \delta)$ if and only if there exists a sequence $j_{1}, \ldots, j_{d}$, possibly empty, such that $\alpha=$ $f_{j_{1}} \circ \cdots \circ f_{j_{d}}(\gamma)$ and $\beta=f_{j_{1}} \circ \cdots \circ f_{j_{d}}(\delta)$.

Reflexivity and transitivity of $\leq$ are obvious. Suppose $(\alpha, \beta) \leq(\gamma, \delta)$ and $(\gamma, \delta) \leq$ $(\alpha, \beta)$. If $(\alpha, \beta) \neq(\gamma, \delta)$, then, by definition of $\leq, \alpha \neq \beta, \gamma \neq \delta$ and there exist indices $j_{1}, \ldots, j_{d}, k_{1}, \ldots, k_{h}$ such that $(\alpha, \beta)=\left(f_{j_{1}} \circ \cdots \circ f_{j_{d}}\right)(\gamma, \delta)$ and $(\gamma, \delta)=$ $\left(g_{k_{1}} \circ \cdots \circ g_{k_{h}}\right)(\alpha, \beta)$. Then $(\alpha, \beta)=\left(f_{j_{1}} \circ \cdots \circ f_{j_{d}} \circ g_{k_{1}} \circ \cdots \circ g_{k_{h}}\right)(\alpha, \beta)$. That is, $t=f_{j_{1}} \circ \cdots \circ f_{j_{d}} \circ g_{k_{1}} \circ \cdots \circ g_{k_{h}}$ has two distinct fixed points, a contradiction. Thus $(\alpha, \beta)=(\gamma, \delta)$ and $\leq$ is also antisymmetric.

We must still show that each $(\alpha, \beta), \alpha \neq \beta$, is bounded below by a diagonal element. If not, then counting shows that there exists a sequence $f_{1}, \ldots, f_{d}$ such that $(\alpha, \beta)=f_{1} \circ \cdots \circ f_{d}(\alpha, \beta)$ and again, $f_{1} \circ \cdots \circ f_{d}$ has two distinct fixed points.

Conversely, suppose such a partial order exists. We must show that each composition of sufficiently many functions $\left\{f_{i}\right\}$ has range consisting of exactly one element. By counting, to this end it suffices to show that for any subset $X \subseteq W_{n}^{k-1}$ with at least two elements and a sufficiently large $r$ the set $f_{1} \circ \cdots \circ f_{r}(X)$ has at least one element less than $X$. To see this take any two distinct elements $\alpha \neq \beta \in X$. Then, by the conditions on $\leq$, eventually $f_{1} \circ \cdots \circ f_{r}(\alpha)=f_{1} \circ \cdots \circ f_{r}(\beta)$, and this does the job. 
Theorem 3.4 and Lemma 4.5 yield the following.

Corollary 4.6. Let $w \in \mathcal{P}_{n}^{k}$. If there exists a partial order on $W_{n}^{k-1} \times W_{n}^{k-1}$ satisfying the conditions of Lemma 4.5, then the endomorphism $\lambda_{w}$ restricts to an automorphism of $\mathcal{D}_{n}$.

The relation used in Lemma 4.5 can be explicitly described as follows. We have that $(\alpha, \beta) \leq(\gamma, \delta)$ if and only if either $\alpha=\gamma$ and $\beta=\delta$, or $\gamma \neq \delta$ and there exist $i_{0}, \ldots, i_{r}$ such that

$$
\begin{array}{cc}
\left(i_{0}, \gamma\right)=\sigma\left(\gamma_{1}, k_{1}\right), & \left(i_{0}, \delta\right)=\sigma\left(\delta_{1}, h_{1}\right) \\
\left(i_{1}, \gamma_{1}\right)=\sigma\left(\gamma_{2}, k_{2}\right), & \left(i_{1}, \delta_{1}\right)=\sigma\left(\delta_{2}, h_{2}\right), \\
\ldots & \ldots \\
\left(i_{r}, \gamma_{r}\right)=\sigma\left(\alpha, k_{r+1}\right), & \left(i_{r}, \delta_{r}\right)=\sigma\left(\beta, h_{r+1}\right)
\end{array}
$$

In order to give an equivalent reformulation of Lemma 4.5 we define inductively a nested sequence of subsets $\Sigma_{m}^{w}$ of $W_{n}^{k-1} \times W_{n}^{k-1}$, as follows:

$$
\begin{aligned}
\Sigma_{0}^{w} & =\left\{(\alpha, \alpha) \mid \alpha \in W_{n}^{k-1}\right\}, \\
\Sigma_{m+1}^{w} & =\left\{(\alpha, \beta) \mid\left(f_{i}(\alpha), f_{i}(\beta)\right) \in \Sigma_{m}^{w}, i=1, \ldots, n\right\} \cup \Sigma_{m}^{w} .
\end{aligned}
$$

We omit an easy proof of the following proposition.

Proposition 4.7. The relation $\leq$ defined by (17) satisfies the conditions of Lemma 4.5 if and only if

$$
\bigcup_{m} \Sigma_{m}^{w}=W_{n}^{k-1} \times W_{n}^{k-1}
$$

4.2. Effect of inner automorphisms. If $w \sim \sigma \in \mathcal{P}_{n}^{k}\left(w=\sum S_{\sigma(\alpha)} S_{\alpha}^{*}\right)$ and $u \sim \phi \in \mathcal{P}_{n}^{k-1}$, then $\operatorname{Ad}(u) \lambda_{w}=\lambda_{\varphi(u) w u^{*}}$ and $\varphi(u) w u^{*} \sim(1 \times \phi) \sigma\left(\phi^{-1} \times 1\right)$.

Let $f_{i}$ and $g_{i}$ be the self-mappings of $W_{n}^{k-1}$ corresponding to $w$ and $\varphi(u) w u^{*}$, respectively, as in (13). Then $(i, \alpha)=\sigma(\beta, m)$ if and only if $(i, \phi(\alpha))=(1 \times$ $\phi) \sigma\left(\phi^{-1} \times 1\right)(\phi(\beta), m)$. Thus $f_{i}(\alpha)=\beta$ if and only if $g_{i}(\phi(\alpha))=\phi(\beta)$. That is,

$$
g_{i}=\phi f_{i} \phi^{-1}, \quad i=1, \ldots, n \text {. }
$$

Consequently, the action of inner automorphisms corresponds to permutation of labels. Thus, combining this observation with Lemma 2.3 we obtain the following.

Proposition 4.8. Suppose that $u, w \in \mathcal{P}_{n}^{k}$ and both $\lambda_{u}$ and $\lambda_{w}$ are automorphisms of $\mathcal{O}_{n}$. If there exists an $i$ such that the tree corresponding to $f_{i}^{u}$ is not isomorphic to the tree of $f_{i}^{w}$ (as a directed tree, no labeling involved), then $\lambda_{u}$ and $\lambda_{w}$ give rise to distinct elements of $\operatorname{Out}\left(\mathcal{O}_{n}\right)$.

Now the following question arises: how many distinct permutations $\tau \in P_{n}^{k}$ give rise to the same collection of labeled trees as $\sigma$ ? The structure and labels on the trees $\left\{f_{1}, \ldots, f_{n}\right\}$ corresponding to $\sigma$ are determined by the identity $(i, \alpha)=$ $\sigma(\beta, m)$, in which $m \in\{1, \ldots, n\}$ can be chosen freely. Thus, simple counting leads to the following. Given any $w \in \mathcal{P}_{n}^{k}$ with corresponding functions $\left\{f_{i}^{w}\right\}$, there are exactly $n !^{n^{k-1}}$ elements $u$ of $\mathcal{P}_{n}^{k}$ yielding identical maps $f_{i}^{u}=f_{i}^{w}$. 
4.3. Lower right corner $[d]$. Now consider the corner $d_{i j}^{w}$ of $a_{i j}^{w}$, where $w \sim \sigma \in$ $P_{n}^{k}$.

The matrix $d_{i j}^{w}$ has a 1 in the $(\alpha, \beta)$ row and the $(\gamma, \delta)$ column if and only if there exists an $m \in\{1, \ldots, n\}$ such that $S_{\alpha} S_{\beta}^{*}=S_{i}^{*} w S_{\gamma} S_{m} S_{m}^{*} S_{\delta}^{*} w^{*} S_{j}$, if and only if there exists some $m$ such that

$$
\begin{aligned}
& (i, \alpha)=\sigma(\gamma, m), \\
& (j, \beta)=\sigma(\delta, m) .
\end{aligned}
$$

Each row of $d_{i j}^{w}$ can have just one 1 or be all 0 's. Summing over all $d_{i j}^{w}, i, j=1, \ldots, n$, each column has a 1 in at most $n$ places (possibly less).

Let $\mathcal{W}_{n}^{k-1}$ be the union of the set of off-diagonal elements of $W_{n}^{k-1} \times W_{n}^{k-1}$ and $\{\dagger\}$, where $\dagger$ is a symbol not in $W_{n}^{k-1} \times W_{n}^{k-1}$. Define mappings $f_{i j}^{w}: \mathcal{W}_{n}^{k-1} \rightarrow \mathcal{W}_{n}^{k-1}$ as

$$
f_{i j}^{w}(\alpha, \beta)=(\gamma, \delta)
$$

if the entry of $d_{i j}^{w}$ in row $(\alpha, \beta)$ and column $(\gamma, \delta)$ is 1 , and as

$$
f_{i j}^{w}(\alpha, \beta)=\dagger
$$

if the $(\alpha, \beta)$ row of $d_{i j}^{w}$ consists of all 0's. In the latter case we think of $f_{i j}^{w}$ as "annihilating" $(\alpha, \beta)$. Also, we put $f_{i j}(\dagger)=\dagger$ for all $i, j$.

Then $d_{i j}^{w} d_{r s}^{w}$ corresponds to $f_{r s}^{w} \circ f_{i j}^{w}$. Again, in the sequel we drop the superscript $w$ when no confusion may arise.

We omit an easy proof of the following proposition.

Lemma 4.9. Let $w \in \mathcal{P}_{n}^{k}$. Then the matrices $\left\{\left[d_{i j}^{w}\right]: i, j=1, \ldots, n\right\}$ generate a nilpotent ring if and only if all sufficiently long composition products of mappings $\left\{f_{i j} \mid i, j=1, \ldots, n\right\}$ have ranges consisting of the single element $\dagger$.

Lemma 4.10. Let $w \in \mathcal{P}_{n}^{k}$. Then the matrices $\left\{\left[d_{i j}^{w}\right]: i, j=1, \ldots, n\right\}$ generate a nilpotent ring if and only if there exists a partial order $\leq$ on $\mathcal{W}_{n}^{k-1}$ such that:

(i) The only minimal element with respect to $\leq$ is $\dagger$.

(ii) For every $(\alpha, \beta) \in \mathcal{W}_{n}^{k-1}$ and all $i, j=1, \ldots, n$,

$$
f_{i j}(\alpha, \beta) \leq(\alpha, \beta) \text {. }
$$

Proof. Suppose that the ring generated by $\left\{\left[d_{i j}^{w}\right]: i, j=1, \ldots, n\right\}$ is nilpotent. Define a binary relation $\leq$ in $\mathcal{W}_{n}^{k-1}$ by (25) and take its reflexive and transitive closure. Suppose for a moment that $(\alpha, \beta) \neq(\gamma, \delta)$ but both $(\alpha, \beta) \leq(\gamma, \delta)$ and $(\gamma, \delta) \leq(\alpha, \beta)$. Then, by definition of $\leq$, there are sequences $i_{1}, \ldots, i_{k}$ and $j_{1}, \ldots j_{k}$ such that $f_{i_{1} j_{1}} \circ \cdots \circ f_{i_{k} j_{k}}(\alpha, \beta)=(\alpha, \beta)$. But then all composition powers of $f_{i_{1} j_{1}} \circ \cdots \circ f_{i_{k} j_{k}}$ have $(\alpha, \beta)$ in their range, a contradiction.

Conversely, suppose that there is a partial order $\leq$ on $\mathcal{W}_{n}^{k-1}$ satisfying condition (ii) above. Then, by counting, each sufficiently long composition product of mappings $\left\{f_{i j}\right\}$ has range consisting of a single element, which is minimal for $\leq$. By (i), this element must be $\dagger$.

Let $w \in \mathcal{P}_{n}^{k}$. We define inductively a nested sequence of subsets $\Psi_{m}^{w}$ of $\mathcal{W}_{n}^{k-1}$, as follows:

$$
\begin{aligned}
\Psi_{0}^{w} & =\{\dagger\}, \\
\Psi_{m+1}^{w} & =\left\{(\alpha, \beta) \in \mathcal{W}_{n}^{k-1} \mid f_{i j}(\alpha, \beta) \in \Psi_{m}^{w}, i, j=1, \ldots, n\right\} \cup\{\dagger\} .
\end{aligned}
$$


We omit an easy proof of the following proposition.

Proposition 4.11. There exists a relation $\leq$ satisfying the conditions of Lemma 4.10 if and only if

$$
\bigcup_{m} \Psi_{m}^{w}=\mathcal{W}_{n}^{k-1}
$$

4.4. A characterization of automorphisms in $\lambda\left(\mathcal{P}_{n}\right)^{-1}$. From Theorem 3.2 Lemma 4.5 and Lemma 4.10 we obtain the following.

Corollary 4.12. Let $w \in \mathcal{P}_{n}^{k}$. Then $\lambda_{w} \in \operatorname{Aut}\left(\mathcal{O}_{n}\right)$ if and only if the following two conditions are satisfied:

(1) There exists a partial order on $W_{n}^{k-1} \times W_{n}^{k-1}$ satisfying the conditions of Lemma 4.5

(2) There exists a partial order on $\mathcal{W}_{n}^{k-1}$ satisfying the conditions of Lemma 4.10 .

\section{Applications of labeled trees to automorphisms of $\mathcal{O}_{2}$}

If $w \in \mathcal{P}_{2}^{k}$, then the labeled trees associated with $f_{1}^{w}$ and $f_{2}^{w}$ have the following properties:

- $\alpha$ receives two edges in $f_{i}^{w}$ if and only if $\alpha$ receives no edges in $f_{3-i}^{w}$;

- $\alpha$ receives one edge in $f_{i}^{w}$ if and only if $\alpha$ receives one edge in $f_{3-i}^{w}$.

It follows that the number of leaves (0-receivers) on both trees are identical and coincide with the number of 2-receivers (including the root) on these trees. In such a case we say that these two (unlabeled) trees are matched.

Given $w \in \mathcal{P}_{2}^{k}$ with corresponding functions $f_{1}^{w}, f_{2}^{w}$ and fixed $i \in\{1,2\}$, we define

$$
G\left(f_{i}^{w}\right):=\left\{\sigma \in P_{2}^{k-1} \mid \sigma f_{i}^{w} \sigma^{-1}=f_{i}^{w}\right\}
$$

and call it the stabilizing group of $f_{i}^{w}$. Let $T$ be the unlabeled rooted tree corresponding to $f_{i}^{w}$. If $\phi \in P_{2}^{k-1}$, then we have $G\left(f_{i}^{w}\right) \cong G\left(\phi f_{i}^{w} \phi^{-1}\right)$, through the map $\sigma \mapsto \phi \sigma \phi^{-1}$. Thus the groups $G\left(f_{i}^{w}\right)$ do not depend on the choice of labels and we have

$$
G\left(f_{i}^{w}\right) \cong \operatorname{Aut}(T),
$$

where $\operatorname{Aut}(T)$ is the automorphism group of the unlabeled rooted tree $T$. Of course, a similar construction can be carried over for any $n$.

5.1. Case of $\mathcal{P}_{2}^{2}$. This case has already been well studied. There are precisely four permutations in $\mathcal{P}_{2}^{2}$ yielding automorphisms of $\mathcal{O}_{2}$. If $F:=S_{1} S_{2}^{*}+S_{2} S_{1}^{*} \in \mathcal{F}_{2}^{1}$ denotes the flip-flop selfadjoint unitary, then the four automorphisms are id, $\lambda_{F}, \operatorname{Ad}(F)$ $=\lambda_{\varphi(F) F}=\lambda_{F \varphi(F)}$, and $\operatorname{Ad}(F) \lambda_{F}=\lambda_{\varphi(F)}$. They form in $\operatorname{Aut}\left(\mathcal{O}_{2}\right)$ a copy of Klein's four-group. In $\operatorname{Out}\left(\mathcal{O}_{2}\right)$, they give $\mathbb{Z}_{2}$ with nontrivial generator the class of Archbold's flip-flop (Bogolubov) automorphism $\lambda_{F}$; see e.g. [14, 15].

Our labeled tree approach gives all these results with almost no effort at all. The only pair of labeled trees satisfying Lemma 4.5 is

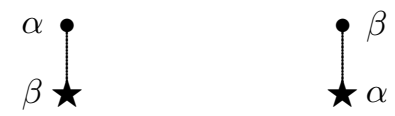


Each is realized by 4 permutations and there are 2 such labelings. Thus there are $2 ! \cdot 2^{2}=2 \cdot 4=8$ permutations in $P_{2}^{2}$ yielding elements of $\operatorname{Aut}\left(\mathcal{D}_{2}\right)$. Of these 8 only 4 give automorphisms of $\mathcal{O}_{2}$.

5.2. Case of $\mathcal{P}_{2}^{3}$. Only two graphs are possible (each self-dual), namely
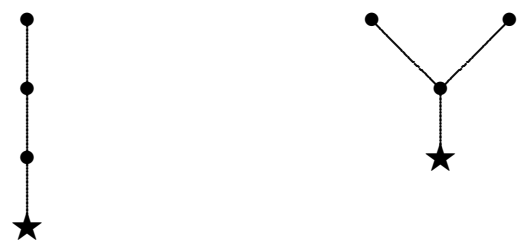

However, there is no labeling of the first graph, which yields a correct partial order $\leq$ on pairs. So only the second graph remains. The only possible labeling satisfying the conditions of Lemma 4.5 is
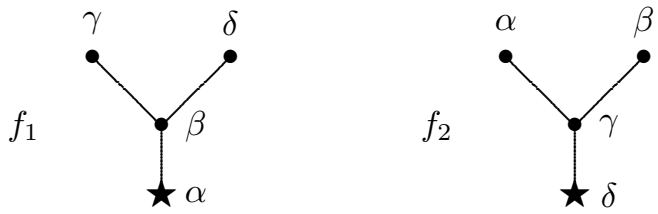

Given a pair of labeled trees as above, there are $2^{4}$ permutations $\sigma \in P_{2}^{3}$ yielding that pair. There are 4 ! possible choices of labels. Hence, there are

$$
4 ! \cdot 2^{4}=24 \cdot 16=324
$$

permutations in $P_{2}^{3}$ satisfying the conditions of Lemma 4.5 and thus yielding elements of $\operatorname{Aut}\left(\mathcal{D}_{2}\right)$.

Then considering 16 permutations giving rise to a fixed labeling, as above, one finds that only two of them satisfy the conditions of Lemma 4.10. Thus, taking into account the action of inner automorphisms corresponding to permutations in $P_{2}^{2}$, we see that there are exactly 48 automorphisms of $\mathcal{O}_{2}$ corresponding to permutations in $P_{2}^{3}$. These are precisely the ones inner equivalent to the identity or the flip-flop. Thus, very surprisingly, among $8 !=40,320$ endomorphisms of $\mathcal{O}_{2}$ from $\lambda\left(\mathcal{P}_{2}^{3}\right)$ the only outer automorphism is the familiar flip-flop. This is in stark contrast with the case of Cuntz algebras $\mathcal{O}_{n}$ with $n \geq 3$, where numerous new outer automorphisms already appear in $\lambda\left(\mathcal{P}_{n}^{2}\right)$ (see the tables in Section 6, below).

Despite the large scale of the problem, our techniques allowed us to obtain these results through easy and straightforward pen and paper calculations. These were further confirmed through a brute force computer calculation.

5.3. Case of $\mathcal{P}_{2}^{4}$. We begin by determining the number of automorphisms in $\lambda\left(\mathcal{P}_{2}^{4}\right)$.

Theorem 5.1. We have

$$
\begin{aligned}
\#\left\{\lambda_{w} \mid w \in \mathcal{P}_{2}^{4} \text { and }\left.\lambda_{w}\right|_{\mathcal{D}_{2}} \in \operatorname{Aut}\left(\mathcal{D}_{2}\right)\right\} & =8 ! \cdot 2^{8} \cdot 17=175,472,640, \\
\#\left\{\lambda_{w} \mid w \in \mathcal{P}_{2}^{4} \text { and } \lambda_{w} \in \operatorname{Aut}\left(\mathcal{O}_{2}\right)\right\} & =8 ! \cdot 14=564,480 .
\end{aligned}
$$

Thus in $\lambda\left(\mathcal{P}_{2}^{4}\right)^{-1}$ there are exactly 14 representatives of distinct inner equivalence classes. 
Proof. There are exactly 23 directed rooted trees (unlabeled) with 8 vertices satisfying our conditions (i.e. each vertex other than the root emits one edge and receives a maximum of 2 edges; the root is a minimal element and receives one edge from a different vertex). A computer calculation shows that there are only 3 matched pairs of such trees admitting labelings satisfying the conditions of Proposition 4.7. These are: $T_{A}-T_{A}, T_{A}-T_{J}$ and $T_{J}-T_{A}$, where $T_{A}$ and $T_{J}$ are as follows (downward oriented):
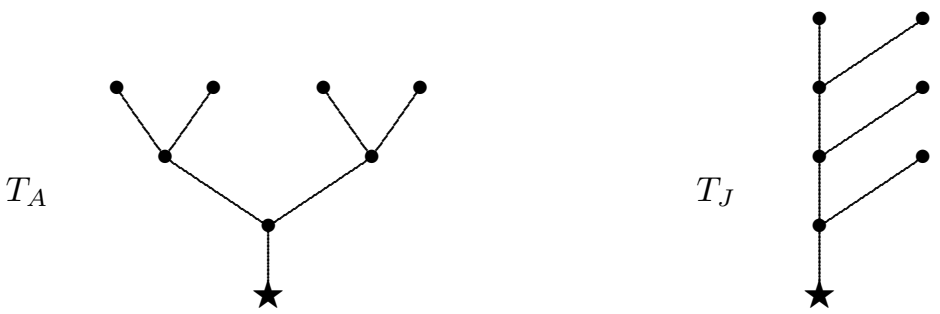

We fix arbitrarily labels on one of the trees in each pair, taking it to be $T_{J}$ in the second and third cases. Then a computer calculation shows the following numbers of labelings of the other tree which satisfy (201): 40 for the pair $T_{A}-T_{A}$ and 12 for each of the other two pairs. The groups of automorphisms of the rooted trees $T_{A}$ and $T_{J}$ have 8 and 2 elements, respectively. Thus, taking into account that each pair of labeled trees under consideration is realized by $2^{8}$ distinct permutations, and factoring in the action of 8 ! inner automorphisms (which permute the labels simultaneously on both trees), we obtain the following number of distinct permutations in $P_{2}^{4}$ giving rise to automorphisms of the diagonal:

$$
2^{8} \cdot \frac{8 !}{\left|\operatorname{Aut}\left(T_{A}\right)\right|} \cdot 40+2 \cdot 2^{8} \cdot \frac{8 !}{\left|\operatorname{Aut}\left(T_{J}\right)\right|} \cdot 12=2^{8} \cdot 8 ! \cdot 17=175,472,640 .
$$

Then a computer calculation shows that among these permutations there are only 8 ! $\cdot 14=564,480$ satisfying (28) and thus yielding automorphisms of $\mathcal{O}_{2}$. Dividing out 8 ! inner automorphisms from level 3 , we finally get 14 inner equivalence classes of automorphisms in $\lambda\left(\mathcal{P}_{2}^{4}\right)^{-1}$.

Our next goal is to describe explicitly representatives of inner equivalence classes from $\lambda\left(\mathcal{P}_{2}^{4}\right)^{-1}$ and to find some infinite subgroups of $\operatorname{Out}\left(\mathcal{O}_{2}\right)$ generated by them.

We begin by considering two permutations $A$ and $B$ of the set $W_{2}^{4}$ given respectively by

$$
\begin{array}{llll}
A(1211)=1211 & A(1212)=1212 & A(1222)=1222 & A(1221)=1221 \\
A(1121)=1121 & A(1122)=1122 & A(1111)=1112 & A(1112)=1111 \\
A(2222)=2111 & A(2221)=2121 & A(2211)=2112 & A(2212)=2122 \\
A(2122)=2222 & A(2121)=2221 & A(2112)=2212 & A(2111)=2211 \\
B(1211)=1211 & B(1212)=1212 & B(1222)=1222 & B(1221)=1221 \\
B(1121)=1121 & B(1122)=1122 & B(1111)=1112 & B(1112)=1111 \\
B(2122)=2111 & B(2121)=2112 & B(2211)=2121 & B(2212)=2122 \\
B(2222)=2212 & B(2221)=2221 & B(2112)=2222 & B(2111)=2211
\end{array}
$$

Note that the first two rows of these two permutations are identical. That is, $A(1 * * *)=B(1 * * *)$. Also, of the first eight arguments, six are fixed points. The labeled trees corresponding to $A$ are: 

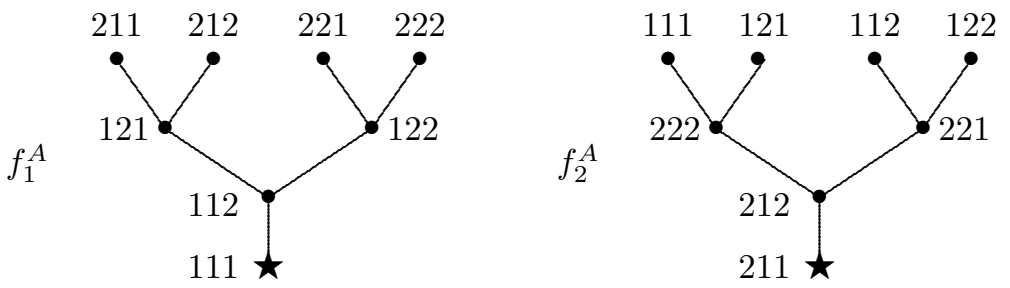

In the sequel, for notational convenience, we equip $W_{2}^{k}$ with the reversed lexicographic order and enumerate its elements as $\left\{1,2, \ldots, 2^{k}\right\}$ accordingly. Then, the permutations $A$ and $B$ above correspond to $A=(1,9)(2,4,10,12,14,16)(6,8)$ and $B=(1,9)(2,4,6,10,16,12,14)$. With a slight abuse of notation we also denote simply by $A$ and $B$ the associated unitaries and by $\lambda_{A}$ and $\lambda_{B}$ the corresponding endomorphisms of $\mathcal{O}_{2}$.

Using Corollary 4.12 one can verify that $\lambda_{A}$ and $\lambda_{B}$ are automorphisms of $\mathcal{O}_{2}$. In fact, these permutations were found through pen and paper calculation based on Corollary 4.12. One checks by a computer calculation based on Section 6.1 that the inverses of the automorphisms $\lambda_{A}$ and $\lambda_{B}$ are induced by unitaries in $\mathcal{P}_{2}^{7}$.

Proposition 5.2. In $\operatorname{Out}\left(\mathcal{O}_{2}\right)$, one has

$$
\lambda_{F} \lambda_{A} \lambda_{F}=\lambda_{A}^{-1}=\lambda_{B} .
$$

Proof. One has $\operatorname{Ad}(z) \lambda_{A} \lambda_{B}=\mathrm{id}$, where $z \in \mathcal{P}_{2}^{6}$ is given by

$$
\begin{aligned}
z \sim & (2,4,8)(3,7,15)(5,13,29)(9,25)(10,12) \\
& (18,20,24)(19,23)(26,28)(34,36,40) \\
& (35,39,47)(37,45)(42,44)(50,52,56)(51,55)(58,60) .
\end{aligned}
$$

Also, one has $\operatorname{Ad}(y) \lambda_{F} \lambda_{A}=\lambda_{B} \lambda_{F}$, where $y \sim(1,3,5,7)(2,4,8) \in P_{2}^{3}$.

For the reader's convenience, in Appendix 8 we provide the action of $\lambda_{A}$ on diagonal projections $P_{\alpha}$ 's with $|\alpha| \leq 5$.

Lemma 5.3. With the above notation, for each word $\tilde{\mu}$ there exist words $\nu_{1}, \nu_{2}$ with $\left|\nu_{i}\right|=|\tilde{\mu}|+1$ such that

$$
\begin{aligned}
& \lambda_{A}\left(P_{\tilde{\mu} 211}\right)=P_{\nu_{1} 211}+P_{\nu_{2} 222}, \\
& \lambda_{A}\left(P_{\tilde{\mu} 212}\right)=P_{\nu_{1} 212}+P_{\nu_{2} 221} .
\end{aligned}
$$

Furthermore, if $\alpha$ is a word which ends neither with 211 nor with 212, then there is a word $\beta$ such that $|\alpha|=|\beta|$ and $\lambda_{A}\left(P_{\alpha}\right)=P_{\beta}$.

Proof. We prove the first claim by induction on $|\tilde{\mu}|$. If $|\tilde{\mu}| \leq 2$ these relations are verified by direct computation. Now let us suppose that $\tilde{\mu}=\left(\mu_{1}, \ldots, \mu_{l}\right)$ and $l \geq 3$. Then

$$
\begin{aligned}
\lambda_{A}\left(P_{\tilde{\mu} 211}\right) & =\lambda_{A}\left(P_{\mu_{1} \ldots \mu_{l} 211}\right)=A^{*} S_{\mu_{1}} \lambda_{A}\left(P_{\mu_{2} \ldots \mu_{l} 211}\right) S_{\mu_{1}}^{*} A \\
& =A^{*} S_{\mu_{1}}\left(P_{\tilde{\nu}_{1} 211}+P_{\tilde{\nu}_{2} 222}\right) S_{\mu_{1}}^{*} A \\
& =A^{*}\left(P_{\mu_{1} \tilde{\nu}_{1} 211}+P_{\mu_{1} \tilde{\nu}_{2} 222}\right) A \\
& =P \ldots 211+P \ldots 222
\end{aligned}
$$


where in the second line we have used the induction hypothesis and in the last line we have used the fact that $\left|\mu_{1} \tilde{\nu}_{1}\right|=\left|\mu_{1} \tilde{\nu}_{2}\right| \geq 4$. The other relation can be handled similarly.

The proof of the second claim proceeds by induction on $|\alpha|$. For $|\alpha| \leq 3$ this follows from the table in Appendix 8 . For the inductive step we notice that there exist two unitaries $u_{1}, u_{2}$ in $\mathcal{F}_{2}^{3}$ such that $\lambda_{A}\left(S_{i}\right)=S_{i} u_{i}, i=1,2$. Thus, we have $\lambda_{A}\left(P_{i \alpha}\right)=\lambda_{A}\left(S_{i}\right) \lambda_{A}\left(P_{\alpha}\right) \lambda_{A}\left(S_{i}\right)^{*}=S_{i} u_{i} P_{\beta} u_{i}^{*} S_{i}^{*}=P_{i \mu}$ for some word $\mu$ with $|\mu|=|\alpha|$.

Proposition 5.4. $\lambda_{A}$ has infinite order in $\operatorname{Out}\left(\mathcal{O}_{2}\right)$.

Proof. It is a consequence of Lemma 5.3 that $\lambda_{A}$ has infinite order in $\operatorname{Aut}\left(\mathcal{O}_{2}\right)$. To see this, fix some $\tilde{\mu}$. If some power of $\lambda_{A}$ were the identity, then, using the relations in Lemma 5.3, one should have that $P_{\tilde{\mu} 211}$ is a sum of subprojections including one of the form $P_{\rho 211}$. But then $P_{\rho 212}$ should also be a subprojection of $P_{\tilde{\mu} 211}$. On the other hand, by the same relations, $P_{\rho 212}$ should be a subprojection of $P_{\tilde{\mu} 212}$ and thus orthogonal to $P_{\tilde{\mu} 211}$, a contradiction.

Now it follows from the implication $(1) \Rightarrow(2)$ of $\left[26\right.$, Theorem 6] that $\lambda_{A}$ has infinite order in $\operatorname{Out}\left(\mathcal{O}_{2}\right)$.

Corollary 5.5. The subgroup of $\operatorname{Out}\left(\mathcal{O}_{2}\right)$ generated by $\lambda_{A}$ and $\lambda_{F}$ is isomorphic to the infinite dihedral group $\mathbb{Z} \rtimes \mathbb{Z}_{2}$.

Let $J$ be a transposition in $P_{2}^{4}$ which exchanges 2112 with 2212 (and fixes all other elements of $\left.W_{2}^{4}\right)$ :

$$
J(2112)=2212 \text { and } J(2212)=2112 .
$$

The labeled trees corresponding to $J$ are:
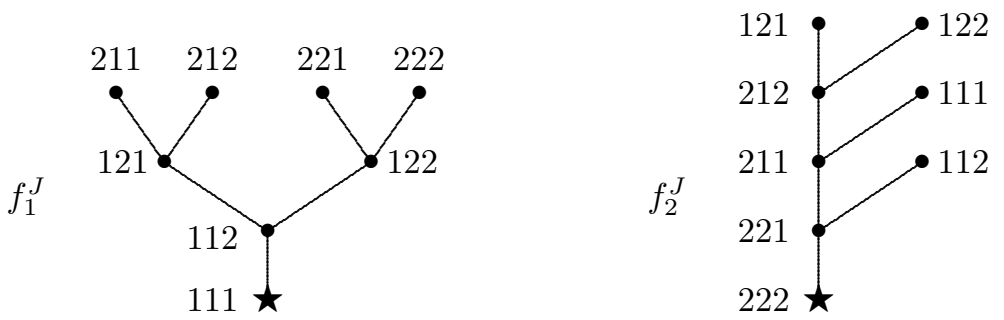

With a slight abuse of notation, we denote by $J$ the associated unitary and by $\lambda_{J}$ the corresponding endomorphism of $\mathcal{O}_{2}$. One checks that

$$
\lambda_{J}^{2}=\mathrm{id} .
$$

Clearly (see Example 4.4), the two trees corresponding to the identity in $P_{2}^{4}$ are both of type $T_{A}$. Likewise, both trees corresponding to the flip-flop $\lambda_{F}$ are also of type $T_{A}$. Since $f_{2}^{J}$ is of type $T_{J} \neq T_{A}$, it follows from Proposition 4.8 that $\lambda_{J}$ is an outer automorphism of $\mathcal{O}_{2}$ not inner equivalent to the flip-flop. Incidentally, outerness of $\lambda_{J}$ can also be derived from [19], since $\lambda_{J}\left(S_{1}\right)=S_{1}$.

Proposition 5.6. The automorphisms $\lambda_{F}$ and $\lambda_{J}$ generate a subgroup of $\operatorname{Out}\left(\mathcal{O}_{2}\right)$ isomorphic to the free product $\mathbb{Z}_{2} * \mathbb{Z}_{2}$.

Proof. The proof is very similar to the argument of Proposition 5.4 and Lemma 5.3. so we only sketch the main idea. 
At first one shows by induction on the word length that for each word $\mu$ there exists a word $\nu$ with $|\nu|=|\mu|+2$ such that

$$
\begin{aligned}
& \lambda_{F} \lambda_{J}\left(P_{\mu 22}\right)=P_{\nu 22}+\sum_{i} P_{\gamma_{i}}, \\
& \lambda_{F} \lambda_{J}\left(P_{\mu 21}\right)=P_{\nu 21}+\sum_{j} P_{\zeta_{j}},
\end{aligned}
$$

with $\gamma_{i}, \zeta_{j}$ words of lengths not greater than $|\nu|+2$. This implies that the automorphism $\lambda_{F} \lambda_{J}$ has infinite order. Consequently, it has an infinite order in $\operatorname{Out}\left(\mathcal{O}_{2}\right)$, and the claim follows.

Let $G$ be a 3 -cycle in $W_{2}^{4}$ such that

$$
G(1112)=1122, \quad G(1122)=1222, \quad \text { and } \quad G(1222)=1112 .
$$

That is, in the shorthand notation, $G=(9,13,15)$. The trees corresponding to $G$ are:
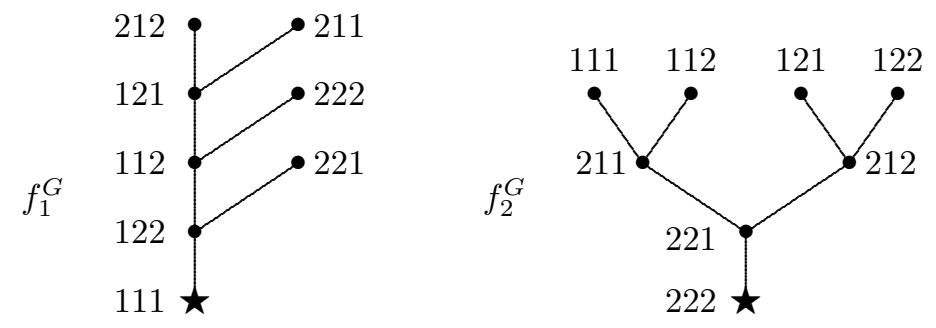

One checks that

$$
\lambda_{G}^{6}=\mathrm{id}
$$

but none of $\lambda_{G}, \lambda_{G}^{2}, \lambda_{G}^{3}$ is inner. Also note that $\lambda_{G}\left(S_{2}\right)=S_{2}$.

Taking into account the results of this subsection and considering the convolution multiplication and Lemma 2.3 (and preferably helped by a computer), one verifies the following theorem.

Theorem 5.7. The following automorphisms give a complete list of representatives of distinct classes in $\operatorname{Out}\left(\mathcal{O}_{2}\right)$ appearing in $\lambda\left(\mathcal{P}_{2}^{4}\right)^{-1}$ :

$$
\begin{aligned}
& \left\{\text { id, } \lambda_{F}\right\}, \\
& \left\{\lambda_{A}, \lambda_{A} \lambda_{F}, \lambda_{F} \lambda_{A}, \lambda_{F} \lambda_{A} \lambda_{F}\right\}, \\
& \left\{\lambda_{J}, \lambda_{J} \lambda_{F}, \lambda_{F} \lambda_{J}, \lambda_{F} \lambda_{J} \lambda_{F}\right\}, \\
& \left\{\lambda_{G}, \lambda_{G} \lambda_{F}, \lambda_{F} \lambda_{G}, \lambda_{F} \lambda_{G} \lambda_{F}\right\} . \\
& \quad \text { 6. TABUlated RESUlts }
\end{aligned}
$$

In this section, we collect our results about automorphisms $\lambda\left(\mathcal{P}_{n}\right)^{-1}$ of the Cuntz algebras in the form of tables. They provide solutions to several enumeration problems.

In the first table, we provide the number $N_{n}^{k}$ of all such automorphisms of $\mathcal{O}_{n}$ at level $k$ (i.e. in $\lambda\left(\mathcal{P}_{n}^{k}\right)^{-1}$ ), for small values of $n$ and $k$. In the second table, we plot the number $C_{n}^{k}$ of classes modulo inner ones (as in Subsection 4.2, only inner perturbations by permutation unitaries are being considered). Of course, we have

$$
N_{n}^{k}=n^{k-1} ! C_{n}^{k} .
$$


The last table contains numbers $s f_{n}^{k}$ of square-free automorphisms in $\lambda\left(\mathcal{P}_{n}^{k}\right)^{-1}$.

\begin{tabular}{|c|c|c|c|c|}
\hline \multirow{5}{*}{$N_{n}^{k}$} & $k \backslash n$ & 2 & 3 & 4 \\
\hline & 1 & 2 & 6 & 24 \\
\hline & 2 & 4 & 576 & $5,771,520$ \\
\hline & 3 & 48 & & \\
\hline & 4 & 564,480 & & \\
\hline
\end{tabular}

\begin{tabular}{|c|c|c|c|c|}
\hline \multirow{5}{*}{$C_{n}^{k}:$} & $k \backslash n$ & 2 & 3 & 4 \\
\hline & 1 & 2 & 6 & 24 \\
\hline & 2 & 2 & 96 & 240,480 \\
\hline & 3 & 2 & & \\
\hline & 4 & 14 & & \\
\hline
\end{tabular}

\begin{tabular}{|c|c|c|c|}
\hline$k \backslash n$ & 2 & 3 & 4 \\
\hline $\bar{~} 1$ & 2 & 4 & 10 \\
\hline 2 & 4 & 52 & 2,032 \\
\hline 3 & 20 & & \\
\hline 4 & 1,548 & & \\
\hline
\end{tabular}

These figures have been obtained through a combination of all the techniques developed in this article and large scale computer calculations. To give the reader an idea of the scale of the problem and the difficulties involved, let us just mention that computation of $N_{4}^{2}$ (and thus $C_{4}^{2}$ ) took about 70 processor days.

\section{Concluding Remarks}

If $n \geq 3$, then the image of $\lambda\left(\mathcal{P}_{n}^{2}\right)^{-1}$ in $\operatorname{Out}\left(\mathcal{O}_{n}\right)$ contains $\mathbb{Z}_{3} * \mathbb{Z}_{2}$ (see [26) and thus it is nonamenable. In the case of $\mathcal{O}_{2}$ we still do not know if the group $\lambda\left(\mathcal{P}_{n}\right)^{-1}$ (and its image in $\operatorname{Out}\left(\mathcal{O}_{2}\right)$ ) is amenable or not. It would be interesting to find the lowest level $k$ (if any) for which $\lambda\left(\mathcal{P}_{2}^{k}\right)^{-1}$ is nonamenable. Our results show that $k$ must be at least 4 , and this question can perhaps be settled by determining the group generated by $\lambda\left(\mathcal{P}_{2}^{4}\right)^{-1}$.

Going beyond automorphisms of $\mathcal{O}_{n}$ preserving the UHF-subalgebra, one may pose the question if any aspects of the theory developed in the present article can be extended to $\lambda\left(\mathcal{S}_{n}\right)^{-1}$. This is certainly far from obvious and undoubtedly a very challenging task. Even the inner part of $\lambda\left(\mathcal{S}_{n}\right)^{-1}$, that is, the group $\mathcal{S}_{n}$ itself, is nonamenable and has a very complicated structure. In fact, in the case of $\mathcal{O}_{2}$, it contains a copy of $\mathbb{Z}_{3} * \mathbb{Z}_{2}$ whose action on the diagonal results in the crossed product isomorphic to $\mathcal{O}_{2}$ 25]. Finding a criterion of invertibility of endomorphisms from $\lambda\left(\mathcal{S}_{n}\right)$ should certainly be regarded as an important first step.

Our labeled tree approach allows for the relatively easy construction of certain special automorphisms of the diagonal $\mathcal{D}_{n}$, which in turn gives rise to dynamical systems on the Cantor set. They certainly deserve further investigations. In particular, a question arises if they may result in minimal dynamical systems. Also, their relation with other better known classes of symbolic dynamical systems is worth elucidation. 
Going beyond the Cuntz algebras, it is natural to expect that parts of our analysis may be extended to more general graph $C^{*}$-algebras. Every graph algebra admits a gauge action of the circle group whose fixed point algebra is $A F$ (e.g. see [23]). For a large class of graph algebras, a Cartan subalgebra with a totally disconnected spectrum is contained in this core $A F$-subalgebra. Then, for such graph algebras, one should be able to say much about the automorphisms preserving both the core $A F$ and the Cartan subalgebra along the lines of [6], 26] and the present article.

\section{ApPENDIX}

\begin{tabular}{|c|c|c|c|}
\hline$P_{\alpha}$ & $\lambda_{A}\left(P_{\alpha}\right)$ & $\lambda_{G}\left(P_{\alpha}\right)$ & $\lambda_{J}\left(P_{\alpha}\right)$ \\
\hline$P_{1}$ & $P_{1}$ & $P_{1}$ & $P_{1}$ \\
\hline$P_{2}$ & $P_{2}$ & $P_{2}$ & $P_{2}$ \\
\hline$P_{11}$ & $P_{11}$ & $P_{111}+P_{1121}+P_{1222}$ & $P_{11}$ \\
\hline$P_{12}$ & $P_{12}$ & $P_{1122}+P_{121}+P_{1221}$ & $P_{12}$ \\
\hline$P_{21}$ & $P_{22}$ & $P_{21}$ & $P_{2111}+P_{212}+P_{2212}$ \\
\hline$P_{22}$ & $P_{21}$ & $P_{22}$ & $P_{2112}+P_{2211}+P_{222}$ \\
\hline$P_{111}$ & $P_{111}$ & $P_{1111}+P_{12221}+P_{11122}$ & $P_{111}$ \\
\hline$P_{112}$ & $P_{112}$ & $P_{11121}+P_{1121}+P_{12222}$ & $P_{112}$ \\
\hline$P_{121}$ & $P_{122}$ & $P_{121}$ & $P_{12111}+P_{1212}+P_{12212}$ \\
\hline$P_{122}$ & $P_{212}$ & $P_{1122}+P_{1221}$ & $P_{12112}+P_{12211}+P_{1222}$ \\
\hline$P_{211}$ & $\begin{array}{l}P_{2211} \\
\quad+P_{2222}\end{array}$ & $P_{2111}+P_{21121}+P_{21222}$ & $P_{2111}+P_{2212}$ \\
\hline$P_{212}$ & $\begin{array}{l}P_{2212} \\
\quad+P_{2221}\end{array}$ & $P_{21122}+P_{2121}+P_{21221}$ & $P_{212}$ \\
\hline$P_{221}$ & $P_{212}$ & $P_{221}$ & $P_{2112}+P_{22111}+P_{22212}$ \\
\hline$P_{222}$ & $P_{211}$ & $P_{222}$ & $P_{22112}+P_{22211}+P_{2222}$ \\
\hline$P_{1111}$ & $P_{1112}$ & $P_{11111}+P_{111122}+P_{111221}$ & $P_{1111}$ \\
\hline$P_{1112}$ & $P_{1111}$ & $P_{111121}+P_{111222}+P_{12221}$ & $P_{1112}$ \\
\hline$P_{1121}$ & $P_{1122}$ & $P_{1121}$ & $P_{112111}+P_{11212}+P_{112212}$ \\
\hline$P_{1122}$ & $P_{1121}$ & $P_{12222}+P_{11121}$ & $P_{112112}+P_{112211}+P_{11222}$ \\
\hline$P_{1211}$ & $\begin{array}{l}P_{12211} \\
\quad+P_{12222}\end{array}$ & $P_{12111}+P_{121121}+P_{121222}$ & $P_{12111}+P_{12212}$ \\
\hline$P_{1212}$ & $\begin{array}{l}P_{12212} \\
\quad+P_{12221}\end{array}$ & $P_{121122}+P_{12121}+P_{121221}$ & $P_{1212}$ \\
\hline$P_{1221}$ & $P_{1212}$ & $P_{1221}$ & $P_{12112}+P_{122111}+P_{122212}$ \\
\hline$P_{1222}$ & $P_{1211}$ & $P_{1122}$ & $P_{122112}+P_{122211}+P_{12222}$ \\
\hline$P_{2111}$ & $P_{2222}$ & $P_{21111}+P_{211122}+P_{212221}$ & $P_{2111}$ \\
\hline$P_{2112}$ & $P_{2211}$ & $P_{211121}+P_{21121}+P_{212222}$ & $P_{2212}$ \\
\hline$P_{2121}$ & $P_{2212}$ & $P_{2121}$ & $P_{212111}+P_{21212}+P_{212212}$ \\
\hline$P_{2122}$ & $P_{2221}$ & $P_{21122}+P_{21221}$ & $P_{212112}+P_{212211}+P_{21222}$ \\
\hline$P_{2211}$ & $\begin{array}{l}P_{21211} \\
\quad+P_{21222}\end{array}$ & $P_{22111}+P_{221121}+P_{221222}$ & $P_{22111}+P_{22212}$ \\
\hline$P_{2212}$ & $\begin{array}{l}P_{21212} \\
\quad+P_{21221}\end{array}$ & $P_{221122}+P_{22121}+P_{221221}$ & $P_{2112}$ \\
\hline$P_{2221}$ & $P_{2112}$ & $P_{2221}$ & $P_{22112}+P_{222111}+P_{222212}$ \\
\hline$P_{2222}$ & $P_{2111}$ & $P_{2222}$ & $P_{222112}+P_{222211}+P_{22222}$ \\
\hline
\end{tabular}




\begin{tabular}{|c|c|c|c|}
\hline$P_{\alpha}$ & $\lambda_{A}\left(P_{\alpha}\right)$ & $\lambda_{G}\left(P_{\alpha}\right)$ & $\lambda_{J}\left(P_{\alpha}\right)$ \\
\hline$P_{11111}$ & $P_{11122}$ & $P_{111111}+P_{1111122}+P_{1111221}$ & $\bar{P} P_{11111}$ \\
\hline$P_{11112}$ & $P_{11121}$ & $P_{1111121}+P_{1111222}+P_{111221}$ & $P_{11112}$ \\
\hline$P_{11121}$ & $P_{11112}$ & $P_{12221}$ & $P_{1112111}+P_{111212}+P_{1112212}$ \\
\hline$P_{11122}$ & $P_{11111}$ & $P_{111121}+P_{111222}$ & $P_{1112112}+P_{1112211}+P_{111222}$ \\
\hline$P_{11211}$ & $\begin{array}{l}P_{112211} \\
+P_{112222}\end{array}$ & $P_{112111}+P_{1121121}+P_{1121222}$ & $P_{112111}+P_{112212}$ \\
\hline$P_{11212}$ & $\begin{array}{l}P_{112212} \\
+P_{112221}\end{array}$ & $P_{1121122}+P_{112121}+P_{1121221}$ & $P_{11212}$ \\
\hline$P_{11221}$ & $P_{11212}$ & $P_{11121}$ & $P_{112112}+P_{1122111}+P_{1122212}$ \\
\hline$P_{11222}$ & $P_{11211}$ & $P_{12222}$ & $P_{1122112}+P_{1122211}+P_{112222}$ \\
\hline$P_{12111}$ & $P_{12222}$ & $P_{121111}+P_{1211122}+P_{1212221}$ & $P_{12111}$ \\
\hline$P_{12112}$ & $P_{12211}$ & $P_{1211121}+P_{121121}+P_{1212222}$ & $P_{12212}$ \\
\hline$P_{12121}$ & $P_{12212}$ & $P_{12121}$ & $P_{1212111}+P_{121212}+P_{1212212}$ \\
\hline$P_{12122}$ & $P_{12221}$ & $P_{121122}+P_{121221}$ & $P_{1212112}+P_{1212211}+P_{121222}$ \\
\hline$P_{12211}$ & $\begin{array}{l}P_{121211} \\
+P_{121222}\end{array}$ & $P_{122111}+P_{1221121}+P_{1221222}$ & $P_{122111}+P_{122212}$ \\
\hline$P_{12212}$ & $\begin{array}{l}P_{121212} \\
\quad+P_{121221}\end{array}$ & $P_{1221122}+P_{122121}+P_{1221221}$ & $P_{12112}$ \\
\hline$P_{12221}$ & $P_{12112}$ & $P_{11221}$ & $P_{122112}+P_{1222111}+P_{1222212}$ \\
\hline$P_{12222}$ & $P_{12111}$ & $P_{11222}$ & $P_{1222112}+P_{1222211}+P_{122222}$ \\
\hline$P_{21111}$ & $P_{22222}$ & $P_{211111}+P_{2111122}+P_{2111221}$ & $P_{21111}$ \\
\hline$P_{21112}$ & $P_{22221}$ & $P_{2111121}+P_{2111222}+P_{212221}$ & $P_{21112}$ \\
\hline$P_{21121}$ & $P_{22112}$ & $P_{21121}$ & $P_{2212111}+P_{221212}+P_{2212212}$ \\
\hline$P_{21122}$ & $P_{22111}$ & $P_{212222}+P_{211121}$ & $P_{2212112}+P_{2212211}$ \\
\hline$P_{21211}$ & $\begin{array}{l}P_{221211} \\
+P_{221222}\end{array}$ & $P_{212111}+P_{2121121}+P_{2121222}$ & $P_{212111}+P_{212212}$ \\
\hline$P_{21212}$ & $\begin{array}{l}P_{221212} \\
+P_{221221}\end{array}$ & $P_{2121122}+P_{212121}+P_{2121221}$ & $P_{21212}$ \\
\hline$P_{21221}$ & $P_{22212}$ & $P_{21221}$ & $P_{212112}+P_{2122111}+P_{2122212}$ \\
\hline$P_{21222}$ & $P_{22211}$ & $P_{21122}$ & $P_{2122112}+P_{2122211}+P_{212222}$ \\
\hline$P_{22111}$ & $P_{21222}$ & $P_{221111}+P_{2211122}+P_{2212221}$ & $P_{22111}$ \\
\hline$P_{22112}$ & $P_{21211}$ & $P_{2211121}+P_{221121}+P_{2212222}$ & $P_{22212}$ \\
\hline$P_{22121}$ & $P_{21212}$ & $P_{22121}$ & $P_{2112111}+P_{211212}+P_{2112212}$ \\
\hline$P_{22122}$ & $P_{21221}$ & $P_{221122}+P_{221221}$ & $P_{2112112}+P_{2112211}+P_{211222}$ \\
\hline$P_{22211}$ & $\begin{array}{l}P_{211211} \\
+P_{211222}\end{array}$ & $P_{222111}+P_{2221121}+P_{2221222}$ & $P_{222111}+P_{222212}$ \\
\hline$P_{22212}$ & $\begin{array}{l}P_{211212} \\
\quad+P_{211221}\end{array}$ & $P_{2221122}+P_{222121}+P_{2221221}$ & $P_{22112}$ \\
\hline$P_{22221}$ & $P_{21112}$ & $P_{22221}$ & $P_{222112}+P_{2222111}+P_{2222212}$ \\
\hline$P_{22222}$ & $P_{21111}$ & $P_{22222}$ & $P_{2222112}+P_{2222211}+P_{222222}$ \\
\hline
\end{tabular}




\section{ACKNOWLEDGEMENTS}

We owe a great debt of gratitude to Dr. Jason Kimberley of Newcastle for his invaluable support with all the computer calculations, which were performed on Magma software [2].

\section{REFERENCES}

[1] R. J. Archbold, On the 'flip-flop' automorphism of $C^{*}\left(S_{1}, S_{2}\right)$, Quart. J. Math. Oxford Ser. (2) 30 (1979), 129-132. MR534827 (80e:47038)

[2] W. Bosma, J. Cannon, and C. Playoust, The Magma algebra system. I. The user language, J. Symbolic Comput., 24 (1997), 235-265. MR 1484478

[3] R. Conti and F. Fidaleo, Braided endomorphisms of Cuntz algebras, Math. Scand. 87 (2000), 93-114. MR 1776967 (2003g:46065)

[4] R. Conti and C. Pinzari, Remarks on endomorphisms of Cuntz algebras, J. Funct. Anal. 142 (1996), 369-405. MR1423039 (98e:46065)

[5] J. Cuntz, Simple $C^{*}$-algebras generated by isometries, Commun. Math. Phys. 57 (1977), 173-185. MR0467330 (57:7189)

[6] J. Cuntz, Automorphisms of certain simple $C^{*}$-algebras, in Quantum fields-algebras, processes, ed. L. Streit, Springer, 1980, pp. 187-196. MR601811 (82d:46089)

[7] J. Cuntz and W. Krieger, A class of $C^{*}$-algebras and topological Markov chains, Invent. Math. 56 (1980), 251-268. MR561974 (82f:46073a)

[8] R. Gohm, A probabilistic index for completely positive maps and an application, J. Operator Theory 54 (2005), 339-361. MR2186358 (2006h:46061)

[9] A. Hopenwasser, J. R. Peters and S. C. Power, Subalgebras of graph $C^{*}$-algebras, New York J. Math. 11 (2005), 351-386. MR2188247 (2007b:47189)

[10] M. Izumi, Subalgebras of infinite $C^{*}$-algebras with finite Watatani indices. I. Cuntz algebras, Commun. Math. Phys. 155 (1993), 157-182. MR1228532 (94e:46104)

[11] M. Izumi, Finite group actions on $C^{*}$-algebras with the Rohlin property. I, Duke Math. J. 122 (2004), 233-280. MR 2053753 (2005a:46142)

[12] V. F. R. Jones, On a family of almost commuting endomorphisms, J. Funct. Anal. 122 (1994), no. 1, 84-90. MR1274584 (95c:46106)

[13] V. F. R. Jones and V. S. Sunder, Introduction to subfactors, London Math. Soc. Lecture Note Ser. 234, Cambridge University Press, Cambridge, 1997. MR.1473221 (98h:46067)

[14] K. Kawamura, Polynomial endomorphisms of the Cuntz algebras arising from permutations. I. General theory, Lett. Math. Phys. 71 (2005), 149-158. MR.2134694 (2006e:46062)

[15] K. Kawamura, Branching laws for polynomial endomorphisms of Cuntz algebras arising from permutations, Lett. Math. Phys. 77 (2006), 111-126. MR2251300 (2008c:46080)

[16] A. Kishimoto and A. Kumjian, Crossed products of Cuntz algebras by quasi-free automorphisms, in 'Operator algebras and their applications' (Waterloo, 1994/1995), 173-192, Fields Inst. Commun. 13, Amer. Math. Soc., Providence, 1997. MR1424962 (98h:46076)

[17] R. Longo, A duality for Hopf algebras and for subfactors. I, Commun. Math. Phys. 159 (1994), 133-150. MR:1257245 (95h:46097)

[18] K. Matsumoto, Orbit equivalence of topological Markov shifts and Cuntz-Krieger algebras, Pacific J. Math. 246 (2010), 199-225. MR2645883

[19] K. Matsumoto and J. Tomiyama, Outer automorphisms of Cuntz algebras, Bull. London Math. Soc. 25 (1993), 64-66. MR1190366 (94a:46089)

[20] H. Matui, Classification of outer actions of $\mathbb{Z}^{N}$ on $\mathcal{O}_{2}$, Adv. Math. 217 (2008), 2872-2896. MR2397470 (2009h:46133)

[21] S. C. Power, Homology for operator algebras, III. Partial isometry homotopy and triangular algebras, New York J. Math. 4 (1998), 35-56. MR1609023 (99g:47108)

$[22]$ S. C. Power, Subalgebras of graph $C^{*}$-algebras, Lecture Notes for the Summer School Course at WOAT 2006, Lisbon, 1-5 September 2006.

[23] I. Raeburn, Graph algebras, CBMS Regional Conf. Series in Math. 103, Amer. Math. Soc., Providence, 2005. MR2135030 (2005k:46141)

[24] M. Rørdam, A short proof of Elliott's theorem: $\mathcal{O}_{2} \otimes \mathcal{O}_{2} \cong \mathcal{O}_{2}$, C. R. Math. Rep. Acad. Sci. Canada 16 (1994), 31-36. MR1276341 (95d:46064) 
[25] J. Spielberg, Free-product groups, Cuntz-Krieger algebras, and covariant maps, Internat. J. Math. 2 (1991), 457-476. MR.1113572 (92j:46120)

[26] W. Szymański, On localized automorphisms of the Cuntz algebras which preserve the diagonal subalgebra, in 'New Development of Operator Algebras', R.I.M.S. Kôkyûroku 1587 (2008), 109-115.

Department of Mathematics, School of Mathematical and Physical Sciences, University of Newcastle, Callaghan, NSW 2308, Australia

Current address: Department of Mathematics, University of Rome 2 Tor Vergata, Via della Ricerca Scientifica, 00133 Rome, Italy

E-mail address: conti@mat.uniroma2.it

Department of Mathematics, School of Mathematical and Physical Sciences, University of Newcastle, Callaghan, NSW 2308, Australia

Current address: Department of Mathematics and Computer Science, The University of Southern Denmark, Campusvej 55, DK-5230 Odense M, Denmark

E-mail address: szymanski@imada.sdu.dk 